\title{
Lattice Boltzmann modeling of heat conduction enhancement by colloidal nanoparticle deposition in microporous structures
}

\author{
Feifei Qin $\odot,{ }^{1,2, *}$ Jianlin Zhao, ${ }^{1}$ Qinjun Kang $\odot,{ }^{3}$ Thomas Brunschwiler, ${ }^{4}$ Dominique Derome, ${ }^{5}$ and Jan Carmeliet ${ }^{1}$ \\ ${ }^{1}$ Chair of Building Physics, Department of Mechanical and Process Engineering, \\ ETH Zürich (Swiss Federal Institute of Technology in Zürich), Zürich 8092, Switzerland \\ ${ }^{2}$ Laboratory of Multiscale Studies in Building Physics, Empa (Swiss Federal Laboratories for Materials Science and Technology), \\ Dübendorf 8600, Switzerland \\ ${ }^{3}$ Earth and Environment Sciences Division (EES-16), Los Alamos National Laboratory (LANL), Los Alamos, New Mexico 87545, USA \\ ${ }^{4}$ Smart System Integration, IBM Research-Zürich, Saumerstrasse 4, 8803 Rüschlikon, Switzerland \\ ${ }^{5}$ Dep. of Civil and Building Engineering, Université de Sherbrooke, Sherbrooke Qc J1K 2R1 Canada
}

(Received 21 February 2020; accepted 25 January 2021; published 26 February 2021)

\begin{abstract}
Drying of colloidal suspension towards the exploitation of the resultant nanoparticle deposition has been applied in different research and engineering fields. Recent experimental studies have shown that neck-based thermal structure (NTS) by colloidal nanoparticle deposition between microsize filler particle configuration (FPC) can significantly enhance vertical heat conduction in innovative three-dimensional chip stacks [Brunschwiler et al., J. Electron. Packag. 138, 041009 (2016)]. However, an in-depth understanding of the mechanisms of colloidal liquid drying, neck formation, and their influence on heat conduction is still lacking. In this paper, using the lattice Boltzmann method, we model neck formation in FPCs and evaluate the thermal performances of resultant NTSs. The colloidal liquid is found drying continuously from the periphery of the microstructure to its center with a decreasing drying rate. With drying, more necks of smaller size are formed between adjacent filler particles, while fewer necks of larger size are formed between filler particle and the top/bottom plate of the FPCs. The necks, forming critical throats between the filler particles, are found to improve the heat flux significantly, leading to an overall heat conduction enhancement of 2.4 times. In addition, the neck count, size, and distribution as well as the thermal performance of NTSs are found to be similar for three different FPCs at a constant filler particle volume fraction. Our simulation results on neck formation and thermal performances of NTSs are in good agreement with experimental results. This demonstrates that the current lattice Boltzmann models are accurate in modeling drying of colloidal suspension and heat conduction in microporous structures, and have high potentials to study other problems such as surface coating, salt transport, salt crystallization, and food preserving.
\end{abstract}

DOI: 10.1103/PhysRevE.103.023311

\section{INTRODUCTION}

Nanoparticle deposition by drying of colloidal suspension has been applied in different research and engineering fields. For instance, with accurate control of nanoparticle assembly, innovative functional materials with better optical, magnetic, and electrical properties can be produced [1-4]. Colloidal nanoparticle deposition on intended specific surfaces is also applied as ink-jet printing [5,6], or surface coating $[7,8]$. Recently, nanoparticle deposition has been used to enhance vertical heat conduction in innovative three-dimensional (3D) chip stacks to remove the heat generated in computer chips more efficiently [9-11]. In a 3D integrated chip stack, two or more layers of active electronic components are integrated vertically, as shown in Fig. S1 in the Supplemental Material [12]. 3D integration can significantly improve the performance of the overall system, such as alleviating power/signal delivery delay [13], reducing chip volume and cointegrating

*Corresponding author: fqin@ethz.ch heterogeneous components [14]. Heat transport of such systems can be an important issue due to the high power density of $3 \mathrm{D}$ integration as well as the low thermal conduction between the different layers.

In the work of Refs. [9-11], percolating thermal structures [PTSs, Fig. 1(a)] and neck-based thermal structures [NTSs, Fig. 1(b) between two vertical chip layers have been proposed to improve heat conduction in 3D chip stacks. NTS, a multiscale structure containing microfiller particles and necks formed by nanoparticles, is the structure of interest in this paper, due to its better thermal performance. The overall fabrication process of NTS in 3D chip stacks consists of five steps. First, the chips are exposed to an atmosphere at $100{ }^{\circ} \mathrm{C}$ for $1 \mathrm{~h}$ to desorb any water. Then the cavity is filled with microsized filler particles between two chip layers by a centrifugation process. Third, a colloidal suspension is injected into the cavity and allowed to evaporate under a heating source, resulting in the formation of necks between the filler particles by nanoparticle deposition, as shown in Fig. 1(b). Different mixes of colloidal nanoparticles were used and are described below when relevant. Subsequently, a matrix 

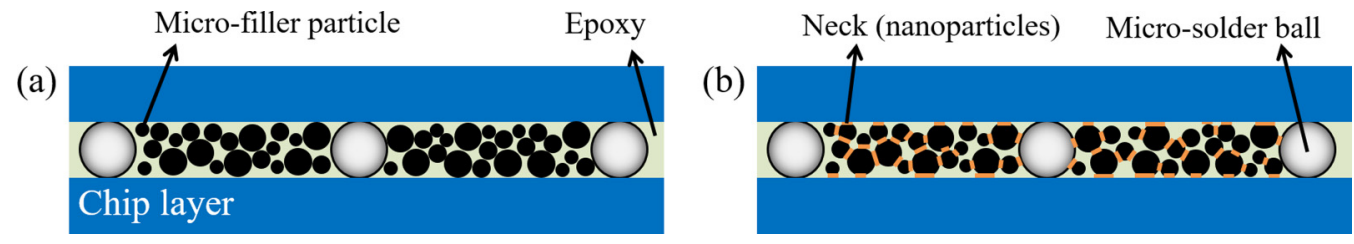

FIG. 1. Illustration of (a) percolating thermal structure and (b) neck-based thermal structure with the necks highlighted in orange color.

material in liquid phase (adhesive polymer like epoxy) is used to fill the cavity by capillary action using an over-pressure. The curing in a nitrogen atmosphere at over-pressure is applied for $12 \mathrm{~h}$ at $120^{\circ} \mathrm{C}$ to fill all pores with the matrix material, removing all the remaining air in the cavity. PTSs are fabricated in a similar way omitting the third step described above. In the experimental study of [9], PTSs and NTSs are reported to increase the heat conduction two- to threefold, compared to state-of-the-art capillary thermal structures. However, an in-depth understanding of the mechanisms of colloidal liquid drying, nanoparticle deposition in the filler particle configuration, and the influence of necks on the heat conduction of NTS is still lacking and needs to be explored systematically. The challenges are threefold. First, drying of colloidal suspension is a multiphysical co-occurring process including coupled two-phase fluid flow, phase change, as well as nanoparticle transport, accumulation and deposition. This process can be influenced by a variety of factors, ranging from the drying temperature or the temperature gradient $[15,16]$, to the liquid and nanoparticle properties [17-20] and drying conditions like forced air convection [21,22]. Second, the process occurring within a microporous structure increases the complexity on nanoparticle transport. For instance, the geometry design [23-25], porosity and surface properties [26-28] can influence the interface pattern of the drying fluid, nanoparticle transport, and the final nanoparticle deposition configuration. Third, understanding the heat conduction in multiscale structures containing micro- and nanoparticles is also very challenging, since the thermal performance of necks formed by nanoparticles is difficult to evaluate. The conjugate heat transport between different components or phases in porous structures also adds to the difficulties [29,30].

In recent years, the lattice Boltzmann method (LBM) has been developed to be an efficient numerical approach to simulate and study complex multiphase [29-32], thermal $[33,34]$, and particulate flows $[35,36]$. Moreover, due to the simplicity in dealing with complex wall boundaries, LBM is abundantly applied in the study of multiphase flows in porous structures [37-39]. For these reasons, here LBM is applied to simulate drying of colloidal suspension in microporous structures. There are two main types of approaches to model particle transport, i.e., Lagrangian method and Eulerian method. In Lagrangian models, the motion of each individual nanoparticle is tracked by solving Langevin equation [40,41] to consider the forces exerted on it as well as its movement. Although Lagrangian models can provide more accurate results considering each individual particle, the computational costs become prohibitively high when a huge number of particles is involved [42]. In Eulerian models, nanoparticles are represented as an "idealized" solute concentration described with a convection diffusion equation $[43,44]$. In this way, the equivalent of a massive number of nanoparticles can be considered easily. For the colloidal suspension under investigation here, we adopt a Eulerian scheme because of its computational efficiency and adequate accuracy, as over millions of particles are present. The Eulerian-type tricoupled hybrid LBM developed in Ref. [43] for two-dimensional (2D) simulation has shown high accuracy and capability in simulating and analyzing two-phase flow, nanoparticle transport and deposition in a thin microporous architecture. In this paper, a tricoupled hybrid LBM is further developed for 3D investigations. In terms of modeling heat conduction in porous structures with the constraint of multicomponent/multiphase conjugate heat transfer, a thermal LBM was proposed in Refs. $[45,46]$ to ensure the continuities of temperature and heat flux at the interfaces. This model has been validated in evaluating the effective thermal conductivity (ETC) of 3D carbon fiber structures in a matrix phase. We note that thermal contact resistance (TCR) [47,48], i.e., the low-conductivity at imperfect interfacial contacts where defects and gaps may be present, can also occur in multicomponent/multiphase systems. However, due to the tiny scale and complex irregular shape of interfaces, the TCR is difficult to be obtained either experimentally or theoretically $[49,50]$. Without an accurate value of the TCR as input parameter, it is impossible to consider its effect numerically. Moreover, even with a given TCR, the accurate heat conduction is very difficult to model, with the existence of a large area of irregular interfaces at micro- and nanoscale. Due to these two reasons, the effect of TCR on heat conduction is not considered in current study. Experimental results in Ref. [9] have reported the non-negligible influences of TCR, which are described in Sec. IV in detail.

In this paper, we apply the aforementioned tricoupled hybrid LBM to study the drying of colloidal suspension in microsized filler particle configurations with neck formation to produce the NTSs. Then we evaluate the thermal performance of the NTSs with the thermal LBM and compare the modeling results with experimental observations. The arrangement of the paper is as follows. The thermal LBM for conjugate heat conduction is introduced in Sec. II and its validation is presented. The tricoupled hybrid LBM for drying of colloidal suspension is described in Sec. III and its validation is provided. Section IV presents results and discussions including neck formation in NTS by drying of colloidal suspension, thermal performance of PTSs and NTSs, as well as comparison with corresponding experimental results. Section V concludes the present work. 


\section{THERMAL LBM FOR CONJUGATE HEAT CONDUCTION}

\section{A. Model description}

To evaluate the thermal performance of the PTS and NTS, we solve the heat transport equation for temperature and heat flux. For a pure thermal conduction with no phase change and no convection, the governing equation is

$$
\left(\rho c_{p}\right)_{p s} \frac{\partial T}{\partial t}=\lambda_{p s} \nabla^{2} T
$$

where the subscript ps represents different phases or components, referring to filler particles, nanoparticle necks or epoxy in this paper. The other parameters $\rho, c_{p}, T, t$, and $\lambda$ are density, specific heat capacity, temperature, time, and thermal conductivity. With the neglect of thermal contact resistance, the temperature and heat flux continuities have to be satisfied at the interfaces between different phases at equilibrium, i.e.,

$$
\begin{aligned}
T_{p s, i}^{\mathrm{int}} & =T_{p s, j}^{\mathrm{int},} \\
\left.\lambda_{p s, i} \frac{\partial T}{\partial \mathbf{n}}\right|_{p s, i} ^{\mathrm{int}} & =\left.\lambda_{p s, j} \frac{\partial T}{\partial \mathbf{n}}\right|_{p s, j} ^{\mathrm{int}},
\end{aligned}
$$

where the superscript "int" represents interfaces and $\mathbf{n}$ is the unit normal vector to the interfaces. Equations (1) and (2) are the governing equations and constraints that describe the multicomponent/multiphase conjugate heat transfer problem.

As mentioned in the introduction, the governing equations are solved by the thermal LBM proposed in Refs. $[45,46]$. The evolution equation of the thermal LBM is given as

$$
g_{i}\left(\mathbf{x}+\mathbf{v}_{i} \delta t, t+\delta t\right)-g_{i}(\mathbf{x}, t)=-\frac{1}{\tau_{p s}}\left[g_{i}(\mathbf{x}, t)-g_{i}^{e q}(\mathbf{x}, t)\right],
$$

where $\mathbf{x}$ is the location vector, $t$ is the time, $\delta t$ is the time step, $g_{i}^{e q}$ is the equilibrium distribution of $g_{i}, \mathbf{v}_{i}, i=0, \ldots, Q-1$ is the discrete velocities, and $\tau_{p s}$ is the relaxation time. For a 3D 15-velocity (D3Q15) LBM, the equilibrium distribution $g_{i}^{e q}$, velocity vectors $\mathbf{v}_{i}$ and relaxation time $\tau_{p s}$ are

$$
\begin{aligned}
& g_{i}^{e q}= \begin{cases}0, & i=0 \\
T / 9, & i=1-6, \\
T / 24, & i=7-14\end{cases} \\
& \mathbf{v}_{i}= \begin{cases}(0,0,0), & i=0 \\
( \pm 1,0,0) c,(0, \pm 1,0) c,(0,0, \pm 1) c, & i=1-6 \\
( \pm 1, \pm 1, \pm 1) c, & i=7-14\end{cases}
\end{aligned}
$$

and

$$
\tau_{p s}=\frac{9}{5} \frac{\lambda_{p s}}{\left(\rho c_{p}\right)_{p s} c^{2} \delta t}+0.5
$$

where $c$ is a pseudo sound speed whose value can take any positive value theoretically only to ensure the value of $\tau_{p s}$ within $(0.5,2)$ [46]. Note that the volume thermal capacities $\left(\rho c_{p}\right)_{p s}$ of different phases/components have to be set identical according to the conjugate heat transfer effect [51]. With solving the LBM in Eq. (3), the temperature $T$ and heat flux $q$ (a) Serial mode
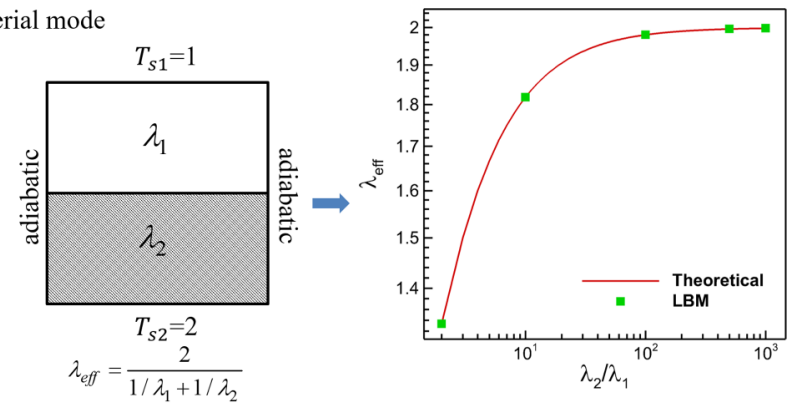

(b) Parallel mode
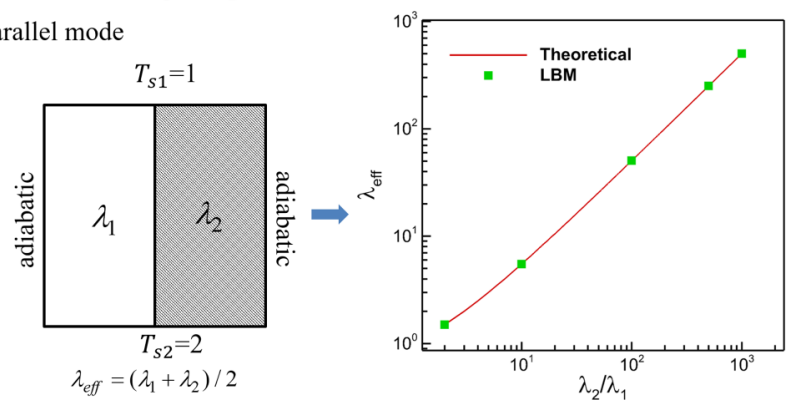

FIG. 2. Validation of the thermal LBM by the evaluation of the effective thermal conductivity (ETC, $\lambda_{\text {eff }}$ ) of two basic dualcomponent systems at various thermal conductivity ratio in (a) serial and (b) parallel modes.

can be calculated as

$$
\begin{aligned}
T & =\sum_{i} g_{i} \\
q & =\left(\rho c_{p}\right)_{p s}\left(\sum_{i} \mathbf{v}_{i} g_{i}\right) \frac{\tau_{p s}-0.5}{\tau_{p s}} .
\end{aligned}
$$

To simplify the computations, we set $\left(\rho c_{p}\right)_{p s}=1$ for all the different phases/components in our simulations. After the temperature and heat flux are solved, the effective thermal conductivity $\lambda_{\text {eff }}$ can be determined by

$$
\lambda_{\mathrm{eff}}=\frac{L \int q d A}{\Delta T \int d A},
$$

where $q$ is the steady state heat flux through the cross section $d A$ for a temperature difference $\Delta T$ over the distance of $L$.

\section{B. Model validation}

The thermal LBM is validated by comparing the ETC of the thermal LBM to theoretical solutions for two basic dualcomponent systems [45]. In the serial mode, the heat flow goes through material 1 then through material 2, while, in the parallel mode, heat flows in the two materials in parallel, as shown in Fig. 2. The 2D simulation setups are the same for both modes, i.e., the temperatures at the top and bottom surfaces are $T_{s 1}=1$ and $T_{s 2}=2$, respectively, while the left and right sides are adiabatic. The ratio of the thermal conductivities of two materials $\lambda_{2} / \lambda_{1}$ ranges from 2 to 1000 (with $\lambda_{1}=1$ ). Figure 2 illustrates that the ETC of serial model increases with $\lambda_{2} / \lambda_{1}$ reaching a plateau value of 2.0 , while the ETC of the parallel model rises linearly with $\lambda_{2} / \lambda_{1}$. Perfect agreement is found between LBM simulation (green squares) and theoretical solution (red lines) for both models, showing 
that the thermal LBM is accurate in evaluating ETC, even with a high ratio of thermal conductivities.

\section{TRICOUPLED HYBRID LBM FOR DRYING OF COLLOIDAL SUSPENSION}

As introduced, the tricoupled hybrid LBM [43,44] is applied here to study the drying of colloidal suspension and nanoparticle deposition in microporous structures. The tricoupled model is built up in the following way. First, an entropic multiple relaxation-time multirange pseudopotential two-phase LBM (EMRT-MP LBM) [29] for isothermal twophase flow is coupled to an extended temperature equation [52] for simulating nonisothermal/quasiisothermal liquid drying (T-EMRT-MP LBM). Then the coupled model is further coupled with a modified convection diffusion equation to handle particle transport and deposition for drying of colloidal suspension (tricoupled hybrid LBM). With this tricoupled model, we are able to study the drying of colloidal suspension with different liquid properties and nanoparticle concentration under various temperatures. The models are briefly summarized as below.

\section{A. EMRT-MP LBM}

For isothermal two-phase flow without phase change, the LBM equation for the populations of discrete velocities that incorporates the external force is

$$
\begin{aligned}
f_{i}(\mathbf{x} & \left.+\mathbf{v}_{i} \delta t, t+\delta t\right) \\
\quad & =f_{i}^{\prime} \equiv(1-\beta) f_{i}(\mathbf{x}, t)+\beta f_{i}^{\operatorname{mirr}}(\mathbf{x}, t)+F_{i},
\end{aligned}
$$

where the equilibrium density populations $f_{i}^{e q}$ maximize the entropy $S[f]=-\sum_{i=1}^{Q} f_{i} \ln \left(\frac{f_{i}}{W_{i}}\right)$ under fixed density and momentum $\rho=\sum_{i=1}^{Q} f_{i}^{e q}, \rho \mathbf{u}=\sum_{i=1}^{Q} \mathbf{v}_{i} f_{i}^{e q}$ while $W_{i}$ are the lattice weights [53]. In D3Q27 LBM, the lattice weights are $W_{0}=\frac{8}{27}, W_{1-6}=\frac{2}{27}, W_{7-18}=\frac{1}{54}, W_{19-26}=\frac{1}{216}$. The liquid kinematic viscosity $v$ is determined by $v=c_{s}^{2}\left(\frac{1}{2 \beta}-\frac{1}{2}\right)$, where $c_{s}=\delta x /(\sqrt{3} \delta t)$ is the lattice speed of sound and lattice units of $\delta x=\delta t=1$ are used with a lattice speed of $c=1$. $\beta$ is a free parameter to adjust the fluid viscosity. The mirror state population $f_{i}^{\text {mirr }}$ is constructed at each lattice site and time step from the entropy maximization of the summarized postcollision population $f_{i}^{\prime}$ by relaxing high-order moments properly [54]. The $f_{i}^{\text {mirr }}$ used in this paper is the same as that in Ref. [29].

The fluid-fluid cohesive force $\mathbf{F}_{c}$ for phase separation and fluid-solid adhesive force $\mathbf{F}_{w}$ for various wettability are incorporated in the last term of Eq. (9) as $F_{i}=f_{i}^{e q}(\rho, \mathbf{u}+\Delta \mathbf{u})-$ $f_{i}^{e q}(\rho, \mathbf{u})$. The velocity increment is $\Delta \mathbf{u}=\mathbf{F} \delta t / \rho$ with the total force $\mathbf{F}=\mathbf{F}_{c}+\mathbf{F}_{w}$. The real velocity of the fluid including the force term is $\mathbf{u}_{f}=\mathbf{u}+\Delta \mathbf{u} / 2$. To make the surface tension tunable, the multirange pseudopotential cohesive force is applied [55]:

$$
\mathbf{F}_{c}=-\psi(\mathbf{x}) \sum_{i=1}^{Q} w\left(\left|\mathbf{v}_{i}\right|^{2}\right)\left[G_{1} \psi\left(\mathbf{x}+\mathbf{v}_{i}\right)+G_{2} \psi\left(\mathbf{x}+2 \mathbf{v}_{i}\right)\right] \mathbf{v}_{i},
$$

where the interaction potential is $\psi=$ $\sqrt{2\left(P_{\mathrm{EoS}}-\rho c_{s}^{2}\right) /\left(G_{1}+2 G_{2}\right) c^{2}}$ and $G_{1}, G_{2}$ are the tuning coefficients for realizing different surface tensions. $P_{\text {EoS }}$ is the equation of state (EoS), and here we adopt the CarnahanStarling $\operatorname{EoS} P_{\operatorname{EoS}}=\rho R T \frac{1+b \rho / 4+(b \rho / 4)^{2}-(b \rho / 4)^{3}}{(1-b \rho / 4)^{3}}-a \rho^{2}$ with the attraction and repulsion parameters $a=0.4963 R^{2} T_{c}^{2} / p_{c}$ and $b=0.18727 R T_{c} / p_{c}$ [56]. $R$ is the gas constant and $T_{c}$ and $p_{c}$ represent the critical temperature and pressure, respectively. In this paper, the parameters are set as $a=1, b=4$ and $R=1$. In this paper, $G_{1}=-1$ and $G_{2}=0$ are set to obtain a surface tension of $\sigma=1.05 \times 10^{-2}$ in lattice units. The fluid-solid adhesive force is represented as

$$
\begin{aligned}
\mathbf{F}_{w}= & -\psi(\mathbf{x}) \sum_{i=1}^{Q} w\left(\left|\mathbf{v}_{i}\right|^{2}\right)\left[G_{1} \psi\left(\rho_{w}\right) I\left(\mathbf{x}+\mathbf{v}_{i}\right)\right. \\
& \left.+G_{2} \psi\left(\rho_{w}\right) I\left(\mathbf{x}+2 \mathbf{v}_{i}\right)\right] \mathbf{v}_{i},
\end{aligned}
$$

where $I$ is the indicator function that equals unity at solid nodes and zero at fluid nodes, $\rho_{w}$ is the parameter to determine surface wettability and $w\left(\left|\mathbf{v}_{i}\right|^{2}\right)$ are the weights in $\mathbf{F}_{c}$ and $\mathbf{F}_{w}$. The contact angle from around $0^{\circ}$ to $180^{\circ}$ can be realized with the selection of $\rho_{w}$ from liquid density $\rho_{l}$ to vapor density $\rho_{v}$ [29]. For D3Q27 lattices, the weights are $w(1)=\frac{1}{6}, w(2)=\frac{1}{12}$ and $w(3)=0$. With this EMRT-MP LBM, isothermal two-phase flow can be accurately simulated in a large range of fluid viscosity with tunable surface tension [29].

\section{B. T-EMRT-MP LBM}

To model nonisothermal/quasi-isothermal liquid drying with phase change, the extended temperature equation that incorporates heat convection/conduction and latent heat is coupled to EMRT-MP LBM to form the T-EMRT-MP LBM. By neglecting viscous heat dissipation, the extended temperature equation derived from the local balance law of entropy [57] is written as [58]

$$
\partial_{t} T=-\mathbf{u}_{f} \bullet \nabla T+\frac{1}{\rho c_{v}} \nabla \bullet(\lambda \nabla T)-\frac{T}{\rho c_{v}}\left(\frac{\partial P_{\mathrm{EOS}}}{\partial T}\right)_{\rho} \nabla \bullet \mathbf{u}_{f},
$$

where $\rho, \lambda, c_{v}$ are the fluid density, fluid thermal conductivity, and specific heat at constant volume. The first two terms on the right-hand side of Eq. (12) represent heat convection and conduction, respectively, while the last term corresponds to the latent heat for phase change. This equation is solved with the finite difference method with a second-order Runge-Kutta scheme for time discretization $T^{t+\delta t}=T^{t}+\frac{\delta t}{2}\left(h_{1}+h_{2}\right)$ with $h_{1}=F\left(T^{t}\right)$ and $h_{2}=F\left(T^{t}+\delta t * h_{1}\right)$ and $F(T)$ represents the right-hand side of Eq. (12). $\delta t$ is the time step in numerical time discretization. For spatial discretization, isotropic central schemes are employed to evaluate the first-order derivative and the Laplacian, with the mesh the same lattice as used in EMRT-MP LBM to avoid extra interpolations [52].

The two-way coupling between the EMRT-MP LBM and extended temperature equation works as follows. At each time step, flow variables like density, velocity, and pressure are firstly computed by solving EMRT-MP LBM. Then they are plugged into the extended temperature equation to update the temperature field. Finally, the updated temperature is incorporated in the computation of flow field by the equation of state for the next time step. The detailed explanation of 
how evaporation is considered is presented in Ref. [52]. The T-EMRT-MP LBM is capable of simulating liquid drying at a high temperature difference and a large thermal conductivity ratio.

\section{Tricoupled hybrid LBM}

To model the transport and accumulation of a large amount of nanoparticles in the liquid, we apply the Eulerian model with a modified convection diffusion equation by considering nanoparticles as a solute [43]:

$$
\frac{\partial \phi}{\partial t}+\nabla \bullet\left(\phi \mathbf{u}_{p}\right)=\nabla \bullet\left(D_{p} \nabla \phi\right)
$$

where $\phi$ and $\mathbf{u}_{p}$ represent the nanoparticle concentration and transport velocity. Considering the two-phase flow, the $\mathbf{u}_{p}$ is modified as $\mathbf{u}_{p}=\mathbf{u}_{f, m}+\Delta \mathbf{u}_{p}$ from the liquid velocity. Here $\mathbf{u}_{f, m}=\mathbf{u}_{f}$ in liquid and $\mathbf{u}_{f, m}=0$ in vapor since particles are present only within the liquid phase. This component of the nanoparticle velocity takes into account the convective delivery of nanoparticles within the liquid phase. The particle velocity increment $\Delta \mathbf{u}_{p}=\mathbf{F}_{p} \delta t / \rho_{p}$ models the fluid-particle interaction $\mathbf{F}_{p}$ which lets nanoparticles accumulate near the liquid-vapor interface, preventing the unphysical occurrence of nanoparticles flowing into the gas phase during evaporation. The particle concentration $\phi=n_{p} * V_{p} / V_{L}$, is the volume fraction of nanoparticle, where $n_{p}, V_{p}$, and $V_{L}$ are number of particles, single nanoparticle volume, and lattice volume, respectively. The fluid-particle interaction $\mathbf{F}_{p}$ is modeled by making an analogy to the fluid-solid interaction $\mathbf{F}_{w}$ in Eq. (11) while considering nanoparticle size and local concentration:

$$
\begin{aligned}
\mathbf{F}_{p}= & -\phi * C_{s, p} * \psi\left(\rho_{p}\right) \sum_{i=1}^{Q} w\left(\left|\mathbf{v}_{i}\right|^{2}\right)\left[G_{1} \psi^{\prime}\left(\mathbf{x}+\mathbf{v}_{i}\right)\right. \\
& \left.+G_{2} \psi^{\prime}\left(\mathbf{x}+2 \mathbf{v}_{i}\right)\right] \mathbf{v}_{i},
\end{aligned}
$$

where $C_{s, p}, \psi^{\prime}$ are the particle size coefficient and modified potential. In this paper, $C_{s, p}=1$ is set and $\psi^{\prime}=$ $\psi^{\prime}\left(\rho_{p} y(\phi), T\right)$ where $y(\phi)=\frac{\rho_{l}}{\rho_{v}}+\frac{1}{2}\left(1-\frac{\rho_{l}}{\rho_{v}}\right)\left\{1-\tanh \left[\left(\frac{\phi}{2}-\right.\right.\right.$ $A) / B]\}$ with $A=0.54$ and $B=0.09$, as explained in Ref. [43] in detail. We note that, currently, we do not consider the influence of nanoparticle surface wettability but assume the nanoparticles to be fully wetting and immersed in the liquid. In the future, we will improve the current model with considering the nanoparticle contact angle effect.

In Eq. (13), $D_{p}$ is the diffusion coefficient derived using the Stokes-Einstein equation as $D_{p}=k_{B} T /\left(6 \pi \mu_{L} R_{p}\right)$, where $k_{B}$ is the Boltzmann constant, $R_{p}$ is particle radius, $T$ and $\mu_{L}$ are the temperature and viscosity of the liquid, respectively [59]. In the experimental study of Ref. [9], the diameter of large nanoparticle is $200-300 \mathrm{~nm}$ while the water dynamics viscosity is $\mu_{L}=4.656 \times 10^{-4} \mathrm{~N} \bullet \mathrm{s} / \mathrm{m}^{2}$ under the temperature of $T=313.15 \mathrm{~K}$, leading to a diffusion coefficient $D_{p}=$ $1.63 \times 10^{-11} \mathrm{~m}^{2} / \mathrm{s}$. In our simulations, the diffusion coefficient is set to $D_{p}=4.0 \times 10^{-3}$ lattice units. To compare our simulation results with experimental ones, we use the nondimensional Peclet number $\operatorname{Pe}_{p}=\frac{\bar{U} H}{D_{p}}$ to evaluate the dominant mechanism between nanoparticle convection and diffusion. The characteristic velocity of nanoparticle $\bar{U}$ can be repre- sented by the average receding speed of liquid. In experiment and simulation, the speeds are $\bar{U}(\exp ) \approx 2.8 \times 10^{-5} \mathrm{~m} / \mathrm{s}$ and $\bar{U}(\operatorname{sim}) \approx 6.7 \times 10^{-3}$ lattice units. The heights of sample are $H(\exp )=60 \mu \mathrm{m}$ in experiment and $H(\operatorname{sim})=90$ lattices in simulation. As a result, the Pelect numbers for experiment and simulation are $\mathrm{Pe}(\exp )=1.03 \times 10^{2} \gg 1$ and $\mathrm{Pe}(\operatorname{sim})=$ $1.34 \times 10^{2} \gg 1$, respectively, thus these numbers are in the same order of magnitude indicating the same mechanismat play, i.e., that the convection is dominant over diffusion in nanoparticle transport.

Similar to the extended temperature equation, Eq. (13) is also solved by finite difference method with a second-order Runge-Kutta scheme. Moreover, a zero-flux boundary condition is enforced at the solid boundary, to make sure that the total particle mass is conserved. For more details on the zero-flux boundary condition in finite difference method, we refer to Ref. [43], where the simulations are in 2D. In current 3D simulations, the modified weight factors $W_{i, m}$ in Eq. (17) of Ref. [43] are changed to

$$
W_{i, m}= \begin{cases}2 / 27, i \in\{1, \ldots, 6\} & i \in \partial S_{1} \\ 1 / 54, i \in\{7, \ldots, 18\} & i \in \partial S_{1} \\ 1 / 216, i \in\{19, \ldots, 26\} & i \in \partial S_{1} \\ 0, & \text { otherwise }\end{cases}
$$

The summations in Eq. (16) in Ref. [43] are changed from 1 to 8 to 1 to 26 . With the current zero-flux boundary condition, the $99.7 \%$ of total nanoparticle mass is conserved in the simulations in this paper. The two-way coupling between TEMRT-MP LBM and particle transport and deposition model is performed with the particle velocity being modified from the fluid velocity obtained from T-EMRT-MP LBM and the newly formed nanoparticle structures influencing the fluid flow.

\section{Validation of the tricoupled hybrid LBM}

This tricoupled hybrid LBM has already been validated with experimental results by 2D simulation of drying of colloidal suspension in thin microporous architectures in Ref. [43]. Here considering the 3D filler particle configuration, we further validate it with the nanoparticle deposition in a two-filler-particle system. The experimental work in Ref. [9] was conducted at a drying temperature of $60{ }^{\circ} \mathrm{C}(333.15 \mathrm{~K})$ with initial alumina nanoparticle concentration (volume fraction of nanoparticle to its water suspension) of around $4 \%$. With the room temperature at $25^{\circ} \mathrm{C}(298.15 \mathrm{~K})$, the difference between the heating temperature and the environment is $35 \mathrm{~K}$. Given the water critical temperature $\left(T_{c}\right)$ of $647 \mathrm{~K}$, the ratio of the temperature difference to the critical temperature becomes $35 / 647 \approx 0.055$. The sample is treated with oxygen plasma to get a contact angle of around $30 \sim 35^{\circ}$. The liquid properties under this drying temperature are given in Table $S 1$ in the Supplemental Material [12]. In current simulations of drying of colloidal suspension, the initial nanoparticle concentration is set $\phi_{n p}=3.7 \%$ and the contact angle is set $30^{\circ}$, values close to the experimental ones. A liquid-vapor density ratio of $\rho_{l} / \rho_{v} \approx$ $30\left(\rho_{l}=0.33\right)$ is used, under the equilibrium temperature of $T_{e q}=0.75 T_{c}$. The equilibrium temperature is considered as the environment temperature set at the boundaries of the four lateral sides, while the fixed drying temperature 


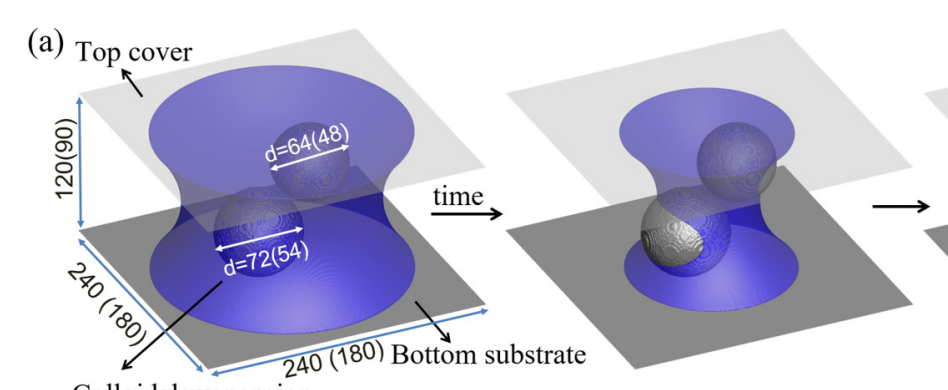

Colloidal suspension

(b)
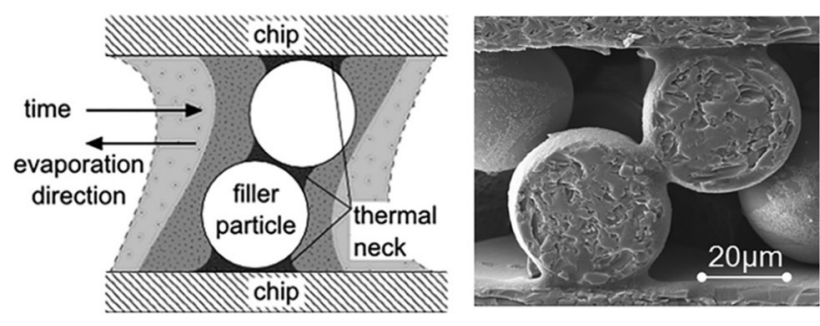

(c)

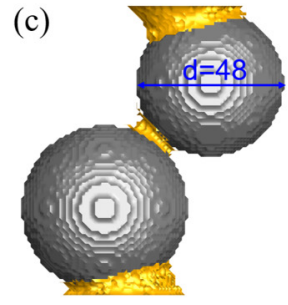

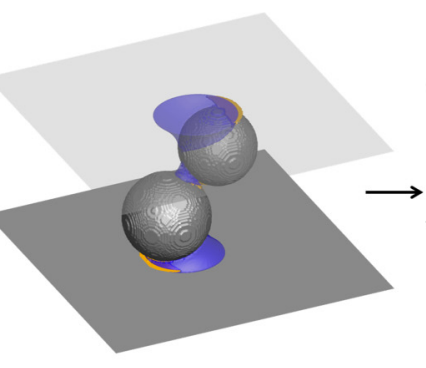

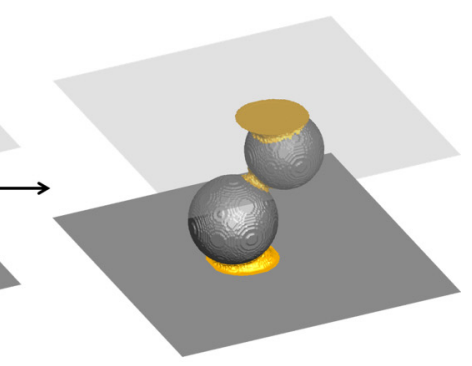

(d)

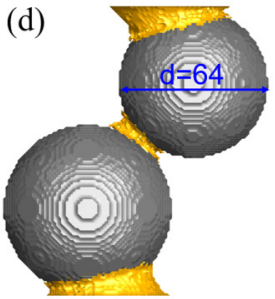

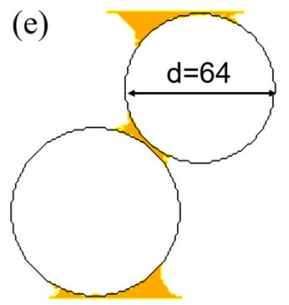

FIG. 3. Validation of the tricoupled hybrid LBM with drying of colloidal suspension and its nanoparticle deposition in a two-filler-particle system. (a) Simulation setup with indications of the two mesh resolutions and simulated process of colloidal liquid drying and nanoparticle deposition in the finer resolution system. (b) Illustration of experimental process of colloidal liquid drying and neck formation by nanoparticle deposition between filler particles from Ref. [9]; (c),(d) Simulated necks (golden) in the coarser and finer resolution systems. (e) Cross section of simulated necks (golden) in finer resolution system. Simulation results are in lattice units while experimental results are in physical units.

$T_{d}=(0.75+0.055) T_{c}=0.805 T_{c}$ is set for the whole system, including the top cover, bottom substrate, colloidal suspension, and filler particles, to ensure temperature conditions similar to those in the experiment. In this way, the drying mechanisms are the same to the ones acting in the experiment. The kinematic viscosities of liquid and vapor are set $v_{l}=v_{v}=0.167$ in lattice units to ensure the same two-phase flow mechanisms as in the experiment. In both the experiment and simulation, drying starts at the lateral sides and continues towards the center. In the experiment, the filler particle diameter ranges from 32 to $36 \mu \mathrm{m}$. Since the nanoparticles deposit at the contact between filler particles and top, bottom plates, and other filler particles, it is critical to model the spherical shape of the filler particle accurately to obtain the realistic deposition configurations. Therefore, two simulations with different resolutions are compared, with the finer resolution of 64 and 72 lattices ( $1 \mu \mathrm{m}=2$ lattices $)$ and a coarser resolution of 48 and 54 lattices ( $1 \mu \mathrm{m}=1.5$ lattices), respectively for the small and large filler particles, as shown in Figs. 3(c) and 3 (d). We note that this small change of resolution is applied for the whole sample and it does not affect the occurring mechanisms and is seen to influence only slightly the nanoparticle deposition configuration. The simulated drying process and neck formation in the two-filler-particle system with higher resolution is shown in Fig. 3(a). Figure 3(b) illustrates the experimental process of drying and deposition, and the final neck formation between the two filler particles in Ref. [9]. The simulated necks with two different resolutions shown in Figs. 3(c) and 3(d) agree both well with the experimental result in terms of the general shapes and locations. Figure 3(e) gives the cross section of the necks in the finer resolution system. The necks show to be with a concave shape, i.e., thin in the middle and thick at the periphery, which is in agreement with the experimental result in Fig. 3(b). The good agreement demonstrates that the tricoupled hybrid LBM is capable of accurately simulating colloidal liquid drying and neck formation in the cavity with microsized filler particles. A resolution of filler particle of 48 lattices is found to be adequate for obtaining accurate simulation results. To illustrate the simulation accuracy, we check the spurious current and compare it with the drying velocity. A simulation is conducted with the same setup but without considering drying. After reaching equilibrium, the spurious current is plotted at the central slice of $x$ direction (Fig. S2a in the Supplemental Material [12]), where we can see that the maximum value is less than $3 \mathrm{e}-3$ lattice units, located at the vapor phase close to the liquid-vapor interface (Fig. S2b in the Supplemental Material). In comparison, the vapor velocity in the drying case here is around 0.16 lattice units (Fig. S2c in the Supplemental Material), which is 53 times higher than the maximum spurious current, indicating the spurious current has negligible influence on our simulation accuracy. We also note that, in the drying of multiple-filler-particle system in Sec. IV below, the drying velocity is not that high, since the pores are quite small not promoting fast drying. We have calculated the average drying velocity as 0.023 lattice units, being eight times of the maximum spurious current. Therefore, our simulation is accurate in the case of drying of a multiple-filler particle system.

\section{RESULTS AND DISCUSSIONS}

This section has four subsections. In Sec. IV A, we simulate the assembly of microsized filler particles in the cavity. As PTSs are fabricated by infiltrating epoxy between filler particles, in Sec. IV B, we evaluate the thermal performance of PTSs in terms of ETC. Subsequently in Sec. IVC, we model the neck formation by drying of colloidal suspension in between the assembled filler particles in order to fabricate NTSs. Finally in Sec. IV D, the thermal performances of the 
formed necks and thus of the NTSs are evaluated. The thermal performances of PTS and NTS, as well as the neck structures are compared with existing experimental results.

\section{A. Assembly of filler particles in the cavity}

In the centrifugation process, i.e., the second step in the fabrication of NTS, the cavity between the two chip layers is filled with microsized particles. This is done by fixing the chip at the periphery of a rotating disk and feeding filler particles from the center of the disk with a nozzle. Upon entry, in this way, the velocity of the filler particle depends on the rotating speed of the disk. Different rotating speed leads to different compaction and FPCs, and thus different filler particle volume fractions. In the work of Ref. [9], the cavity is around $14000 \times 12000 \times 60 \mu \mathrm{m}^{3}$, the diameter of the filler particles is $34 \pm 2 \mu \mathrm{m}$, and the filler particle volume fraction around $47 \%$.

In order to obtain a comparable assembly of filler particle, the centrifugation process is mimicked using the discrete element method, as fully described in Ref. [60]. We use the LIGGGHTS open source code (Version 3.3.1) for simulating the filler particle assembly [61]. The motion for each individual particle is tracked considering the balance of forces and torques, with the detailed explanation in Ref. [61]. To produce an assembly, 82 filler particles are randomly located in a cavity of $2000 \times 240 \times 60 \mu \mathrm{m}^{3}$, more precisely the system counts $9,23,21,21$, and 8 particles with diameter of $32,33,34,35$, and $36 \mu \mathrm{m}$, respectively. The filler particles are given an initial velocity of $0.12 \mathrm{~mm} / \mathrm{s}$ along the length direction, and the simulation time step is $1.5 \times 10^{-4} \mathrm{~s}$. All the faces of cavity are considered as solid walls and the filler particles are smooth and rigid. After 500000 iterations, the filler particles arrive at a static configuration. The simulation is run with eight processors for $2 \mathrm{~min}$. A particle bed of size $240 \times 240 \times 60 \mu \mathrm{m}^{3}$ is generated and employed in this work. Due to the limitation of computation resources, this final system is small compared to the actual chip cavities [9], but care is taken below to ensure that it is employed as an appropriate unit volume element.

Figure 4(a) illustrates the initial random distribution of filler particles with the velocity direction, while Fig. 4(b) illustrates the intermediate state during the assembly process. Figure 4(c) displays the final compact FPC with 82 particles. The compact FPC is extracted as shown in Fig. 4(d), resulting in a particle volume fraction $V_{f p}^{n, t}$, i.e., total filler particle volume $V_{f p}^{t}$ versus cavity volume $V_{c v}$, being $48.8 \%$. This value is very close to the experimental one of $47.0 \%$. Figure 4(e) shows the extracted FPC that is used below for the evaluation of ETC and for the simulation of neck formation by LBM in next subsections. This extraction is required because discrete element method (DEM) tracks the configuration using the center location and diameter of each particle. Such information is not suitable for LBM, which needs the actual geometry, i.e., whether each discretization point lies inside the pore space or not.

To check the influence of filler particle configuration on the thermal performance of resulting PTS and NTS, two additional FPCs with similar filler particle volume fraction $V_{f, t}^{n, t}$ of $48.0 \%$ and $47.1 \%$ are assembled using this method. FPC 1

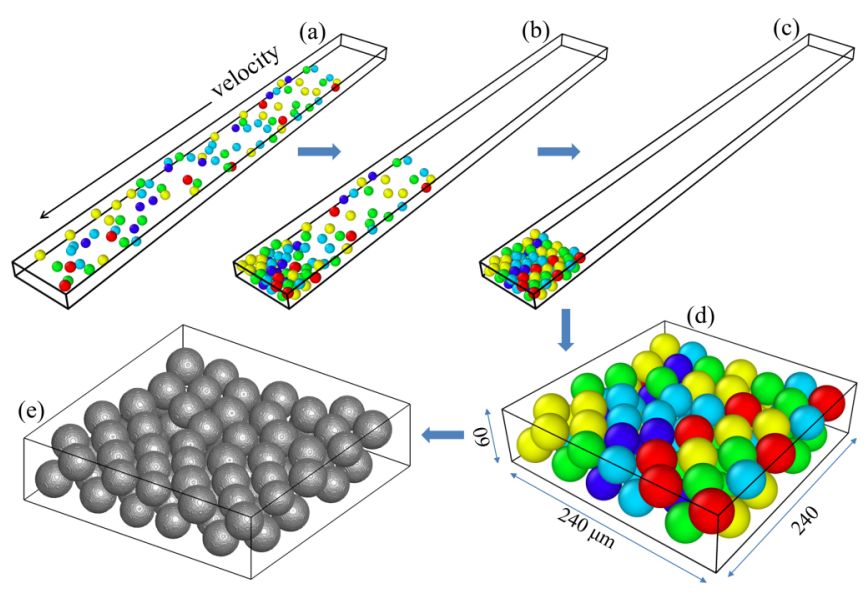

FIG. 4. Simulation of the assembly process of filler particles in the cavity between two chip layers in 3D chip stacks using the discrete element method. (a) Initial random distribution of filler particles. (b) Intermediate state during the assembly process. (c) Final assembled compact filler particle configuration (FPC). (d) Extracted cavity with the volume of compact FPC with dimensions (physical units). (e) Extracted FPC for subsequent LBM simulations.

is shown in Fig. 4 and the additional FPCs are denoted as FPC2 and FPC3, and shown in Fig. S3 in the Supplemental Material. PTS2 contains 79 filler particles with 8, 21, 22, 20, and 8 particles of diameter of $32,33,34,35$, and $36 \mu \mathrm{m}$, respectively. PTS3 contains 80 filler particles with $8,25,24$, 18 , and 5 particles of diameter of $32,33,34,35$, and $36 \mu \mathrm{m}$, respectively.

\section{B. Thermal performance of the PTS}

Using the assembled FPCs and assuming the pores between filler particles completely filled with epoxy, the PTS is numerically produced. The three different FPCs give rise to PTS1 to PTS3, as shown in Fig. 5(a). The thermal performance of the PTSs is evaluated numerically in terms of ETC using the thermal LBM introduced in Sec. II. In the experiment of Ref. [9], the filler particles are alumina with thermal conductivity of $30 \mathrm{~W} / \mathrm{mK}$, while the thermal conductivity of epoxy is $0.21 \mathrm{~W} / \mathrm{mK}$. To calculate the ETC of PTSs, we impose a temperature gradient over the sample from bottom to top. The exact value of the temperature gradient is not important for the calculation, since all the thermal properties of the materials used are independent of temperature. Thus, the temperatures of the bottom substrate and top cover are set to $T_{\text {bot }}=2$ and $T_{\text {top }}=1$. The four lateral sides are set to be adiabatic, guaranteeing no heat loss. The dimensions of the cavities are $360 \times 360 \times 90$ lattice $^{3}$, with the filler particle diameter ranging from 48 to 54 lattices, corresponding to the range from 32 to $36 \mu \mathrm{m}$ in the experiments. After the production of FPC, the diameters of all filler particles are decreased by 1 lattice in simulations to avoid contact between filler particles. This procedure succeeds in approximating the actual situation after capillary filling with epoxy, as, in experiments, it has been observed that the filler particles do not contact with each other directly but are connected by a thin layer of epoxy [9]. With the same preoccupation, the location of top cover and bottom is also shifted by 1 lattice away from the 


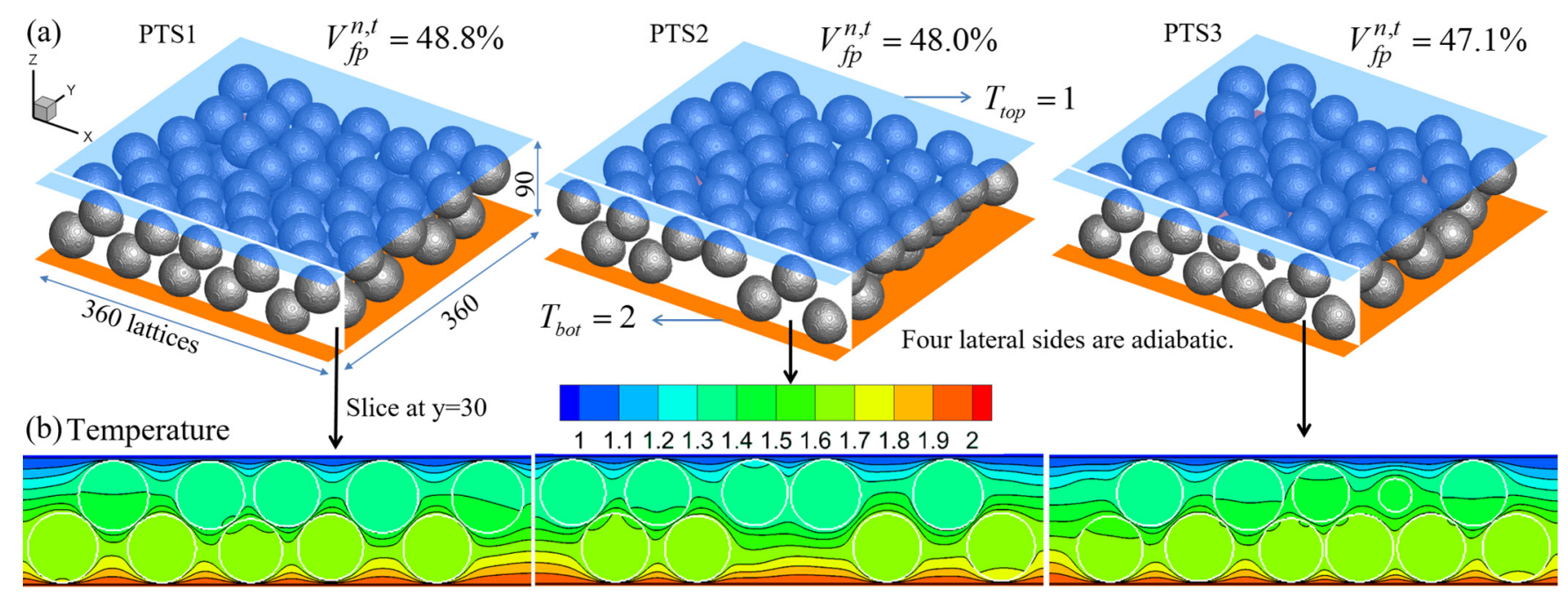

(c) Heat flux

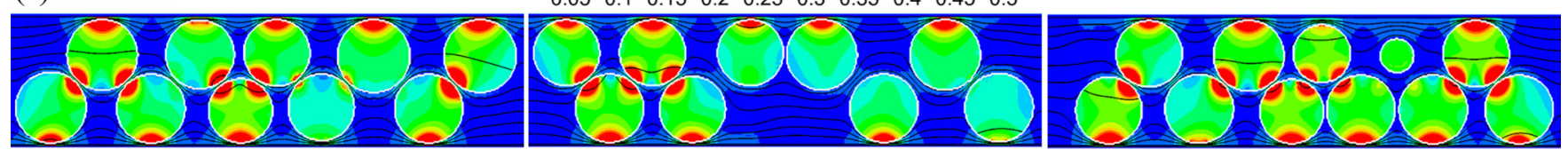

FIG. 5. Evaluation of thermal performance of the percolating thermal structures (PTSs). (a) Simulation setups of three different PTSs with similar filler particle volume fraction $\left(V_{f p}^{n, t}=48.8 \%, 48.0 \%, 47.1 \%\right)$, with the top and bottom temperatures $T_{\text {top }}=1$ and $T_{\text {bot }}=2$ and four lateral sides adiabatic. (b) Temperature distribution with isolines in black at the slice of $y=30$ in the three different PTSs, and white lines indicating boundaries of filler particles. (c) Heat flux contours at the slice of $y=30$ in different PTSs, with black lines indicating temperature isolines and white lines indicating boundaries of filler particles. All variables are in lattice units.

filler particle to avoid contact between filler particle and top cover/bottom substrate.

To verify that the results are mesh independent, a comparison of the ETC of a two-particle system is done at two resolutions: a coarser resolution with filler particles of 48 and 54 lattices, and a finer resolution with ones of 64 and 72 lattices. The results are compared and a $3.7 \%$ difference of ETC indicates that the coarser resolution of filler particle with a diameter of at least 48 lattices is acceptable. Details of the comparison is shown in Fig. S4 in the Supplemental Material.

Figures 5(b) and 5(c) show the temperature and heat flux distribution for the three different PTSs at the slice of 30 lattices in the $y$ direction. The temperature in the alumina filler particles is very uniform, compared to the larger temperature gradient in the epoxy, due to the large thermal conductivity difference between alumina and epoxy (around 143 times larger for alumina). Moreover, the heat flux in the filler particles is much higher than that in the epoxy. At the nearcontact locations, i.e., where filler particles are close to the top cover, bottom substrate or other filler particles, the heat flux is markedly higher. These locations are "critical throats" that determine the amount of heat transported from bottom substrate to top cover. The calculated ETCs of the three PTSs are $\lambda_{\text {eff }}(\mathrm{PTSs})=1.22,1.21,1.19 \mathrm{~W} / \mathrm{mK}$. The small variation of $2.4 \%$ indicates that the centrifugation method used for generating filler particle configurations leads to PTS realizations with similar overall performance, although the local temperature and heat flux are different due to the different distribution of filler particles. Compared with the measured ETC of $1.0 \mathrm{~W} / \mathrm{mK}$ in average in the experiment of Ref. [9], the simulation results overestimate the ETC by around $20 \%$.
The experimental error was estimated to be around $\pm 5.0 \%$, but sources of error are not much discussed in the paper. From the simulation, there are three possible reasons for the discrepancy. First, the size of the current cavity is not as big as that in the experiment, although of equivalent height. Second, the boundary conditions of the four lateral sides are not adiabatic in the experiment, which leads to an additional heat loss to the sides. Third, the thermal contact resistance between the epoxy/filler particles/cover is not considered in the simulations, which is reported to be around $0.3 \mathrm{~W} / \mathrm{mK}$ in Ref. [9]. The latter might be the major cause of discrepancy. This indicates that further work would be necessary to ascertain a proper thermal resistance at the contacts between different materials, but such work is deemed out of the scope of the current paper. Overall, considering this complex fabrication and measuring process, from an engineering point of view, the thermal LBM is quite acceptable in studying the heat transport in such kind of microporous structures, if not with absolute values, at least by comparing performance of different systems.

\section{Neck formation by colloidal nanoparticle deposition in the FPC}

The third step of the fabrication of NTS consists in neck formation by colloidal nanoparticle deposition in the FPC. We simulate this process by the tricoupled hybrid LBM introduced in Sec. III. Taking the first filler particle configuration (FPC1) for illustration, the simulation setup is shown in Fig. 6(a). Except the geometrical information, i.e., the sample size, filler particle number and distribution, all other 

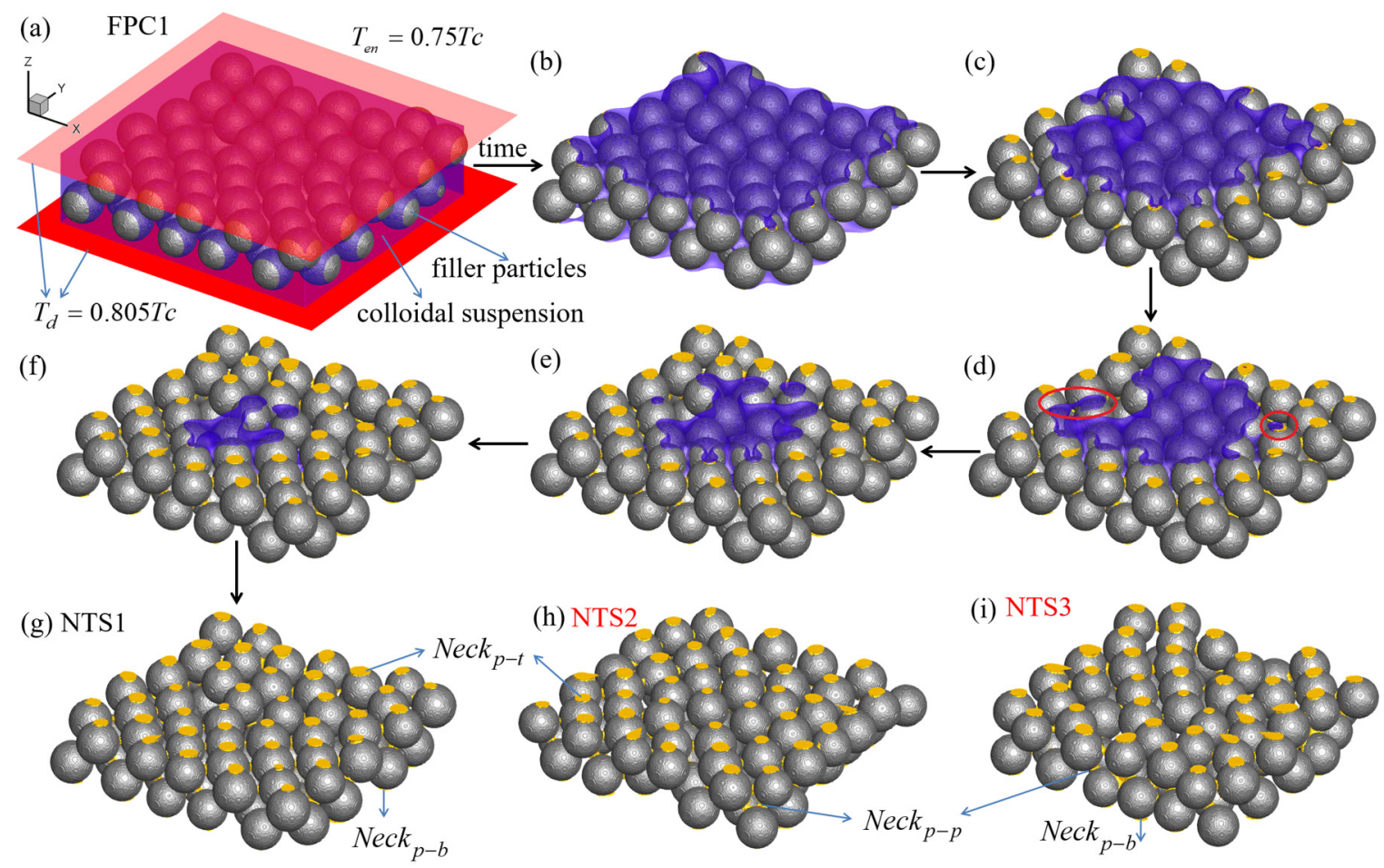

FIG. 6. Drying of colloidal suspension in cavity with filler particle configuration 1 (FPC1), and the resultant neck formation in other two neck-based thermal structures (NTSs). (a) Simulation setup of drying of colloidal suspension in FPC1. (b)-(g) Different stages during the drying process of colloidal liquid in FPC1 and formation of necks by nanoparticle deposition, giving rise to the NTS1. (h) and (i) The resultant neck formation in NTS2 and NTS3.

simulation parameters are set the same as in Sec. III D to ensure the conditions similar to those in the experiment of Ref. [9].

The drying process is shown in Figs. 6(b)-6(g). We can see that the colloidal liquid recedes from the periphery to the center of the FPC. In the experiment of Ref. [9], two drying patterns are observed in sequence. As shown in Fig. S5a in the Supplemental Material, at first, a dendritic pattern is observed where the large pores are invaded from the two open ends (inlet and outlet) to the central part of the system. Afterwards, capillary bridges are formed between adjacent filler particles and between filler particle and top/bottom plates until drying completes. In our simulation, we have observed both the dendritic network [blue liquid in Figs. 6(c)-6(f) and the collapse of capillary bridges [in red eclipses in Fig. 6(d)]. Figure $\mathrm{S} 5 \mathrm{~b}$ in the Supplemental Material gives more illustrations. Since our system $(240 \mathrm{um} \times 240 \mathrm{um} \times 60 \mathrm{um})$ is small compared to the experimental one $(12 \mathrm{~mm} \times 14 \mathrm{~mm} \times 60 \mathrm{um})$, the dendritic network is not as clear as in the experiment. The capillary bridges have a shorter lifetime in our simulation. However, our simulation generally captures well the main mechanisms of colloidal dying and resultant neck formation. To analyze and compare the drying rate for all three FPCs, we use the total drying time $t_{1}$ and initial liquid mass $m_{1}$ of FPC1 as references. The three drying times are close to each other, with a $\pm 2 \%$ difference, as shown in the $x$ axis of Fig. 7. The normalized time during drying is calculated as $t_{N}=t / t_{1}$ and the normalized liquid mass is defined as $m_{N}=m\left(t_{N}\right) / m_{1}$. The normalized drying rate $E p_{N}$ is calculated within each time period of $\Delta t_{N}=0.02$, i.e., $E p_{N}=$
$\left(m_{N}\left(t_{N}+\Delta t_{N}\right)-m_{N}\left(t_{N}\right)\right) / \Delta t_{N}$. The normalized liquid mass in Fig. 7(a) shows a concave shape for all the FPCs, indicating drying becomes slower with time. The evaporation rates in Fig. 7(b) illustrate a decreasing trend with a turning point "P" at around $t_{N}=0.1$. Before this point, the drying rate is high and decreasing quickly; while afterwards, it is lower with a smaller decreasing speed. The explanation is as following. As shown in the inset of Fig. 7(b), the turning point represents the state where the liquid just recedes into the porous system, i.e., the space between the filler particles, from the initial state denoted by the blue-dashed box. From the initial state to the turning point, the liquid interface is completely exposed to the environment connected to the outlet boundary, making it easy for vapor to go out and thus leading to a high drying rate. Afterwards when the drying menisci enter the pores, it is less straightforward for vapor to diffuse out, leading to a smaller drying rate. With the drying interface farther away from the outlet, the drying rate decreases as long as the drying continues. As described above, the local drying finishes after the capillary bridges break. Specifically, it completes at locations with smaller curvature radius, i.e., the "critical throats" between two adjacent filler particles $L_{p-p}$, between filler particle and top cover $L_{p-t}$, and between filler particle and bottom substrate $L_{p-b}$, where the liquid pressures are lower. Concurrently, the necks are formed by nanoparticle depositions at locations $L_{p-p}, L_{p-t}$ and $L_{p-b}$ are denoted as $\mathrm{Neck}_{p-p}$, Neck ${ }_{p-t}$, and $\mathrm{Neck}_{p-b}$, respectively. The formed necks in the filler particle configurations FPC2 and FPC 3 are shown in Figs. 6(h) and 6(i). The deposition configurations have similar features in the three NTSs to be discussed below. 

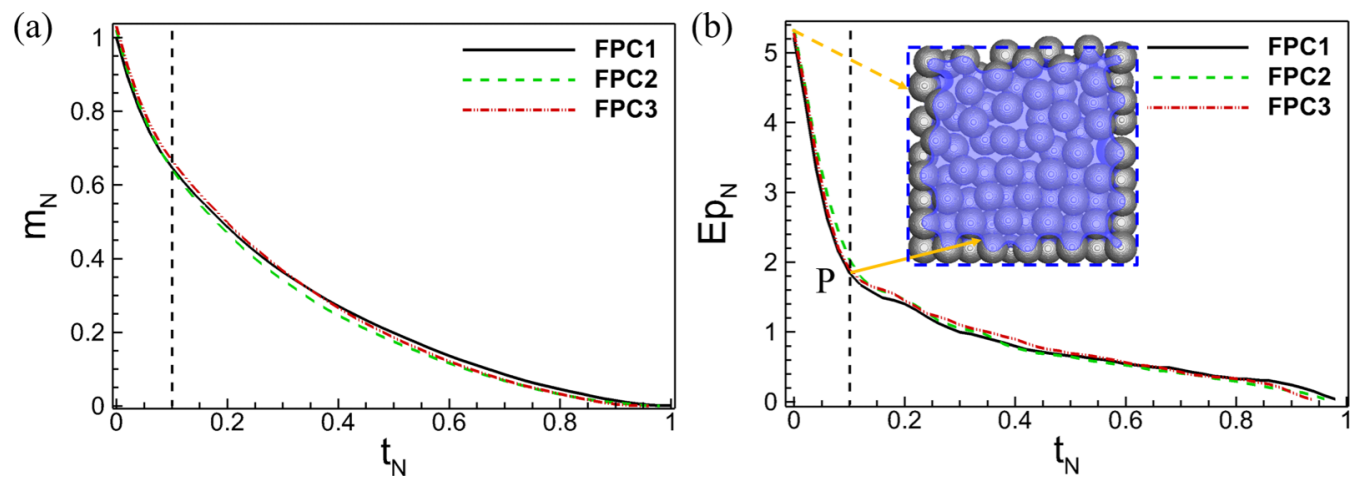

FIG. 7. (a) Normalized colloidal liquid mass and (b) evaporation rate during colloidal liquid drying in three filler particle configurations (FPCs). Inset shows liquid configuration at point $\mathrm{P}$, where the drying rate suddenly decreases since the liquid-vapor interface starts receding into the pores inside the FPC. The dashed blue line shows initial liquid configuration when drying occurs.

In the experiment of Ref. [9], the average diameters of the necks formed against flat surfaces, i.e., necks at the top cover $\left(\mathrm{Neck}_{p-t}\right)$ and bottom substrate $\left(\mathrm{Neck}_{p-b}\right)$ are measured. Taking the slices at the top cover and bottom substrate of the three simulated NTSs and integrating the neck area, the total neck area is obtained. Dividing the total neck area by the neck number at the top cover and substrate, the average area of one neck is obtained. Assuming the neck is circular, the average neck diameter can be obtained. The normalized average diameter $d_{\text {neck }}^{n, a}$ at top and bottom, i.e., ratio of the measured average neck diameter $d_{\text {neck }}^{a}$ to the average filler particle diameter $d_{f p}^{a}, d_{\text {neck }}^{n, a}\left(\operatorname{Neck}_{p-t, p-b}\right)=d_{\text {neck }}^{a} / d_{f p}^{a}$, is found to be around $40 \%$ experimentally and $39.4 \%, 41.7 \%$, and $42.2 \%$, respectively, for the three simulated systems, thus very similar to the experimental result.

In the experiment, it is hard to measure the average diameter of necks between filler particles $\left(\mathrm{Neck}_{p-p}\right)$, due to the difficulty in extracting the irregularly distributed necks, which can be easily damaged when removing the filler particles. Using the simulation results, we can extract and analyze the necks easily. Taking NTS1 as an example, 261 necks are extracted and shown in Fig. 8(a). The dimensions of the necks at the top/bottom $\left(\mathrm{Neck}_{p-t}\right.$, Neck $\left.p-b\right)$ and necks between filler particles $\left(\mathrm{Neck}_{p-p}\right)$ are compiled and analyzed separately. Similar to the normalized average neck diameter $d_{\text {neck }}^{n, a}$, the normalized average neck volume $V_{\text {neck }}^{n, a}$ is defined as the ratio of average neck volume $V_{\text {neck }}^{a}$ to average filler particle volume $V_{f p}^{a}$, i.e., $V_{\text {neck }}^{n, a}=V_{\text {neck }}^{a} / V_{f p}^{a}$. Figure 8(b) shows that, on average, the normalized average neck volume at the top/bottom of 0.79 is around 2.7 times of the neck volume of 0.29 between particles. In counterpart, there are 82 top/bottom necks $\left(\operatorname{Neck}_{p-t, p-b}\right)$ versus 179 interparticle necks $\left(\operatorname{Neck}_{p-p}\right)$. The higher neck volume for top/bottom necks is explained by the higher void fraction near the top and bottom compared to that in the middle, as shown in Fig. 9(a). The larger pore volumes at the top and bottom indicate that more colloidal suspension and thus more nanoparticles are available for local deposition. Similarly, the $d_{\text {neck }}^{n, a}$ of necks at top/bottom is 1.8 times of those between filler particles with a slightly higher variation shown in Fig. 8(c). In Fig. 8(c), the average value of $d_{\text {neck }}^{n, a}$ at top/bottom equals to $44.3 \%$. This value is calculated based on the inertia tensor of the 3D neck structure. Considering the necks having the form of an ellipse, the inertia tensor gives the longest and shortest diameter of the ellipse. The average diameter of the neck is calculated as the square root of the product of long and short diameters obtained from the inertia tensor. This method for determining the average neck diameter is slightly different from the method using the top view of the necks based on a 2D slice. As described above, the value obtained by the $2 \mathrm{D}$ slice method yields an average neck diameter of $39.4 \%$. The $4.9 \%$ deviation between two different methods is caused by an inaccuracy of the calculation of inertia tensor, since the neck structure is very irregular. The small deviation indicates that the postprocessing method yields acceptable results. Figures 9(b)-9(d) compare the three systems from NTS1 to NTS3, in terms of neck count, diameter
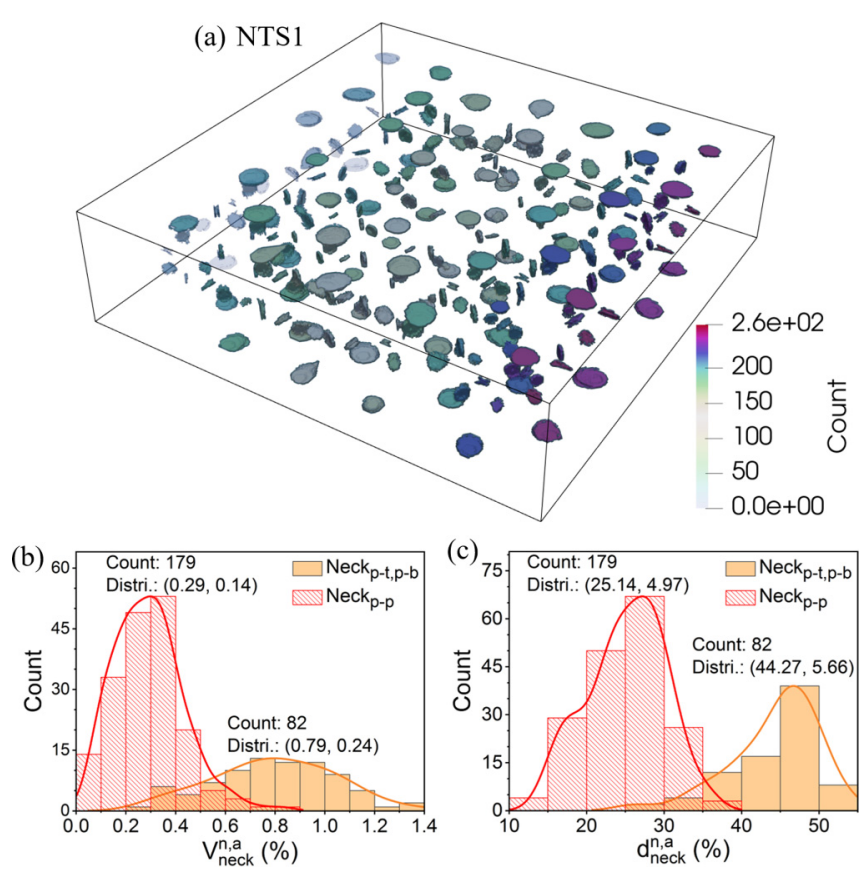

FIG. 8. Analysis of deposited necks in NTS1. (a) NTS1 counts 261 necks, shown here by removing the particles. Histograms of (b) normalized average neck volume $\left(V_{\text {neck }}^{n, a}\right)$ and (c) normalized average neck diameter $\left(d_{\text {neck }}^{n, a}\right)$ of necks between particle and flat substrate and cover $\left(\operatorname{Neck}_{p-t, p-b}\right)$, and between particles $\left(\operatorname{Neck}_{p-p}\right)$, where the "Distri." values indicate mean value and standard deviation. 

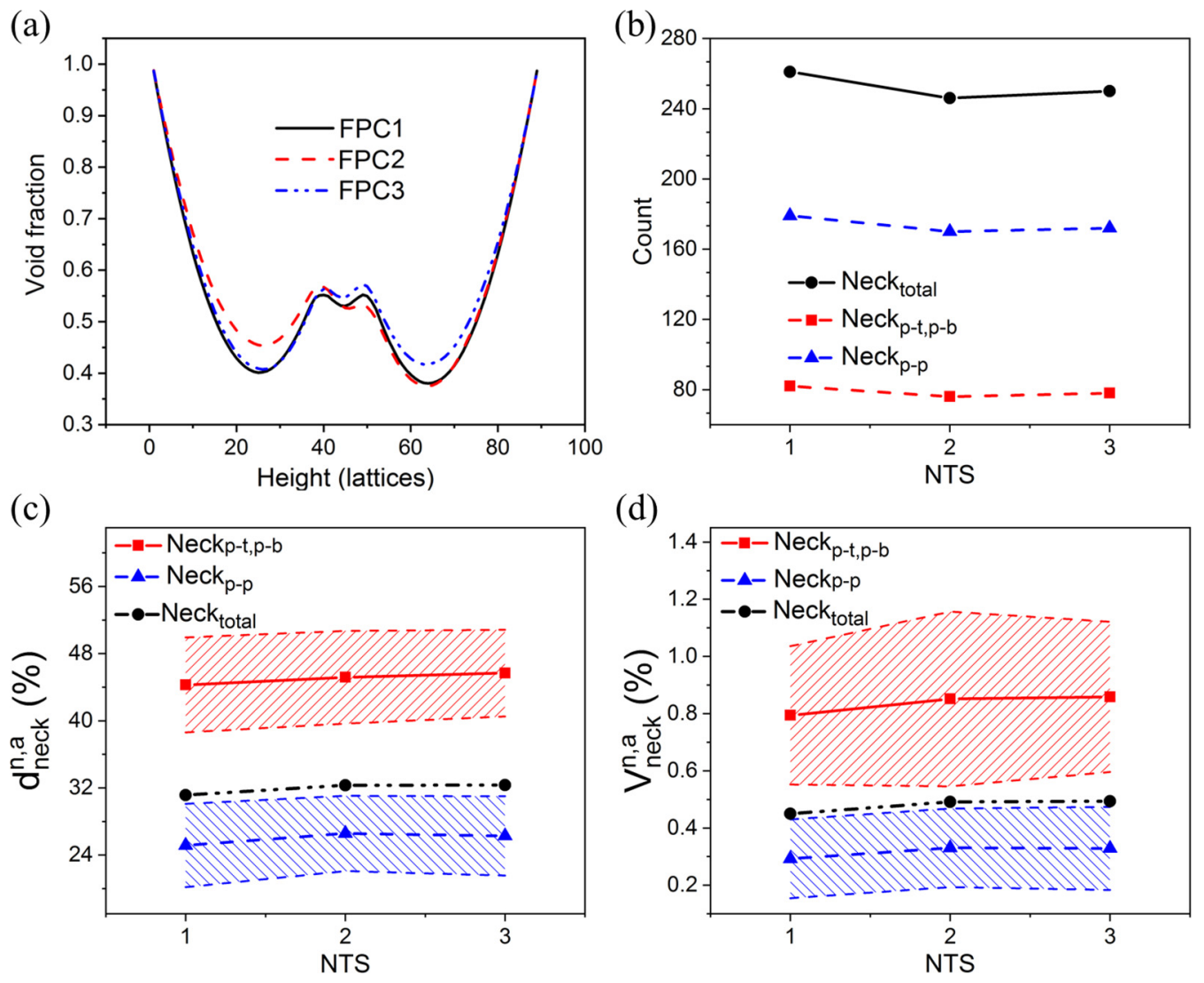

FIG. 9. Comparison of the metrics of three filler particle configurations (FPCs) and the deposited necks in neck-based thermal structures (NTSs). (a) Void fraction over the height of system. (b) Neck counts. (c) Neck diameter. (d) Neck volume.

$d_{\text {neck }}^{n, a}$, and volume $V_{\text {neck }}^{n, a}$. These values barely vary between the different NTSs indicating the DEM-based centrifugation method of assembling filler particles has little influence on the formation of necks and is adequate for engineering applications. Further, the hybrid LBM allows more metrics to be obtained, like the volume of all necks and the diameter of necks between filler particles. We note that the comparison of the diameter of the top/bottom necks between experiments and simulations provides another aspect of validation of the hybrid LBM.

The good agreement on neck location and diameters, in average equal to $40 \%$ of filler particle diameter, between current simulations and experimental results in Ref. [9] is considered. In terms of the simulation conditions, we remind the reader that we aimed to match the relevant parameters with those in the experiment. First, the assembly of filler particles is similar and the resultant volume fractions are approximately the same ( $48.8 \%$ in the simulation and $47 \%$ in experiment). The small discrepancy $(3.8 \%)$ results from a smaller number of filler particles in a smaller simulation domain. Second, the initial nanoparticle concentrations are very close, i.e., 3.7\% in the simulation and $4 \%$ in the experiment. Third, the normalized differences between drying and environment temperatures are the same, i.e., $\left(T_{d}-T_{e n}\right) / T_{c}=0.055$. Fourth, the contact angles are kept almost identical $\left(30 \sim 35^{\circ}\right)$. Due to the limitation of numerical model, it is challenging that all the parameters of liquid properties are identical to those in the experiment. Nevertheless, with the given simulation parameters of fluid density, viscosity, surface tension as well as nanoparticle diffusion coefficient, the important nondimensional numbers that describe fluid flows and mass transport, i.e., liquid Reynolds number $(\Leftarrow 1)$, two-phase capillary number $(\ll 1)$, vapor Peclet number $(\ll 1)$, and nanoparticle Peclet number $(\gg 1)$, are actually in ranges similar to those in the experiment, providing assurance that the same mechanisms are at play. Namely, the liquid flow is laminar (Stokes flow), the capillary force is dominant between liquid and gas over viscous force, the vapor transport is diffusive, and the nanoparticle transport is convective in the liquid. The good agreement between simulation and experimental results including the same nanoparticle deposition location and neck diameters indicates that the choice of those parameter values is reasonable.

We note that the reduction of filler particle diameter by 1 lattice from the original FPC is informed from the final experimental results of NTS in Ref. [9], where the filler particles generally do not contact directly, but are connected by the nanoparticle deposited necks. The neck thickness is measured to reach up to $2 \mu \mathrm{m}$, thus $5.8 \%$ of filler particle diameter of $34 \mu \mathrm{m}$ (Fig. S6 in the Supplemental Material). In our simulations, by decreasing the filler particle diameter by 1 lattice, we obtain an average neck thickness of 1 to 2 lattice, thus $1.9 \%$ to $3.9 \%$ of filler diameter, which is within the thickness range in the experiment. In addition, the diameters of the necks at the top and bottom of the sample are very close to the experimental measurement, i.e., $40 \%$ of filler particle diameter. Decreasing systematically the diameter of the filler 

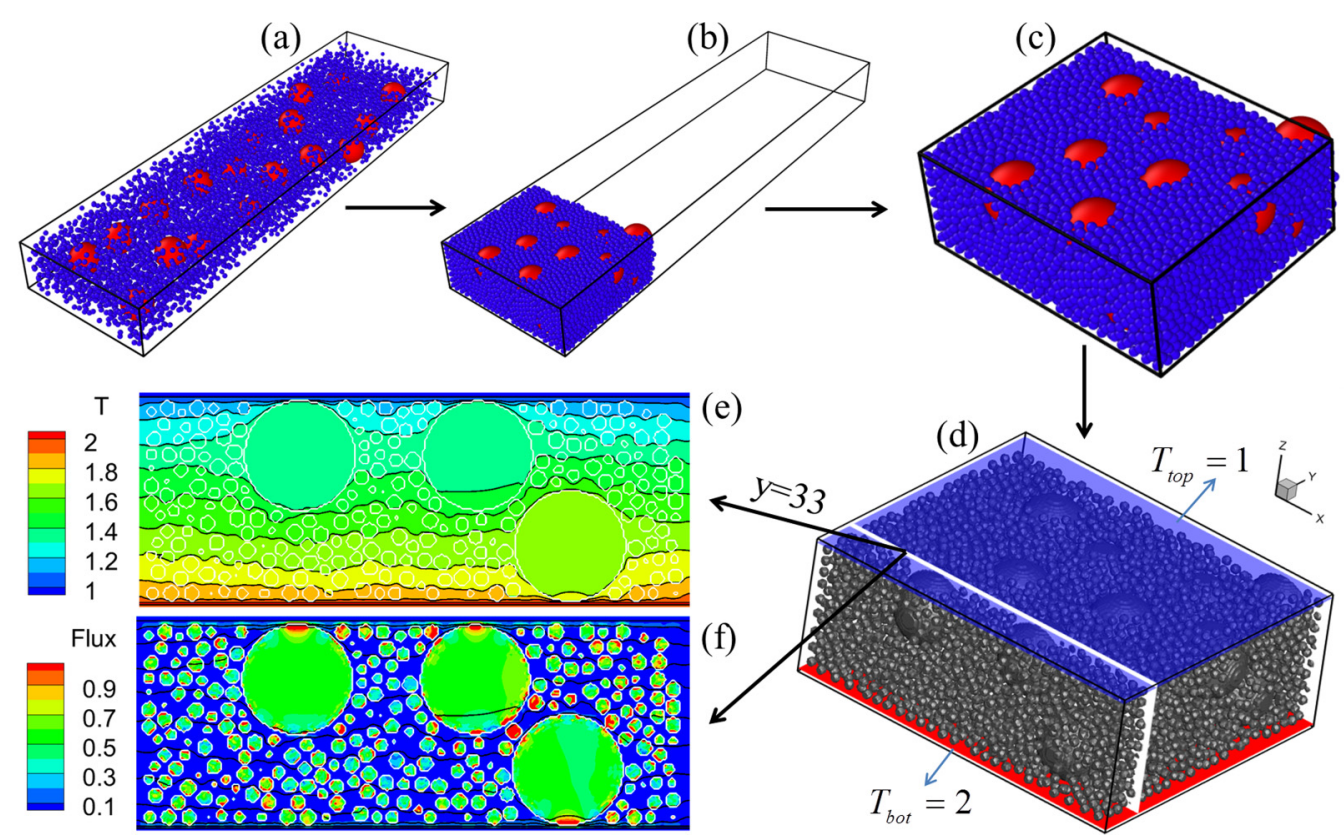

FIG. 10. Calculation of effective thermal conductivity (ETC) of the nanocomposite material making the necks. (a) Distribution of nanoparticles of two different sizes at initial state. (b) Final assembled nanoparticle deposition configuration by discrete element method. (c) Extracted compact nanoparticle deposition configuration. (d) Simulation setup of the system with top cover and bottom substrate temperatures $T_{\text {top }}=1, T_{\text {bot }}=2$ and lateral sides adiabatic. (e) Temperature distribution with temperature isolines in black and nanoparticle outlines in white at the slice of $y=33$. (f) Heat flux distribution with temperature isolines in black and particle outlines in white at the slice of $y=33$. All variables are in lattice units.

particles by 1 lattice is a pragmatic choice and a better way would require acquiring the experimental filler particle gap distribution and incorporating them in the simulation. However, it is experimentally impossible yet to acquire the geometry of the filler particles before colloidal deposition.

\section{Thermal performance of the NTS}

We evaluate the thermal performance of NTS and compare it with PTS, in terms of the ETC. Given the configuration of the systems, a multiscale approach is used, calculating first the ETC of the nanocomposite materials forming the necks and then using this obtained ETC for the global heat conduction evaluation of the NTS, a mixture of micro and nanocomposite materials.

\section{ETC of the neck material}

The ETC of the material forming the necks is first calculated here. The necks are structures made of nanoparticles of two different diameters and infiltrated by epoxy. In the experiment of Ref. [9], the nanoparticles in the colloidal suspension are of two diameters, i.e., $40 \mathrm{~nm}$ (blue) and $250 \mathrm{~nm}$ (red), as shown in Fig. 10(a). The volume ratio of the smaller versus larger nanoparticles is 1.5 . The assembly process of nanoparticles is simulated similarly to the assembly process of the filler particle configuration of PTS in Sec. IV A, as shown in Figs. 10(a)-10(c). The resulting structure shows a good mix of the two particle types, with a nanoparticle volume fraction of $66.9 \%$.

The extracted nanoparticle configuration shown in Fig. 10(d) is employed to calculate the ETC of the neck using the thermal LBM. The method described in Sec. IV B is followed here also. The top cover and bottom substrate temperatures are set as $T_{\text {top }}=1$ and $T_{\text {bot }}=2$ while the four lateral sides are adiabatic. All nanoparticles are alumina with a thermal conductivity of $30 \mathrm{~W} / \mathrm{mK}$, while the void between the particles is filled with epoxy with a thermal conductivity of $0.21 \mathrm{~W} / \mathrm{mK}$. Both the nanoparticles and filler particles are made of alumina due to its high thermal conductivity to enhance the heat conduction. Since alumina is an electrical insulator, it may be applied in computer chip assemblies. The simulated temperature distribution with isolines (black lines) at the slice of $y=33$ lattices is shown in Fig. 10(e). Since most of the nanoparticles are small, the temperature distribution is generally uniform from bottom to top. The flux distribution with temperature isolines (black lines) at the slice of $y=33$ lattices is shown in Fig. 10(f), where the heat flux in the aluminum nanoparticles is much higher than that in the epoxy matrix. With the simulated temperature and flux fields, the ETC of the neck is calculated to be $\lambda_{\text {eff }}($ neck $)=2.38 \mathrm{~W} / \mathrm{mK}$. We note that this material ETC has not been measured experimentally to our knowledge.

\section{ETC of the NTS}

Having determined the ETC of the neck material, we can evaluate the ETC of NTS, similarly to that of PTS, as shown in Fig. 11(a). Figure 11(b) gives the temperature distribution and isolines (in black) at the slice of $y=30$. Figure 11(c) compares the temperature isolines for NTS1 (green) and PTS1 (red). The NTS1 isolines are less dense at the necks, indicating the local temperature gradients are smaller due to improved 


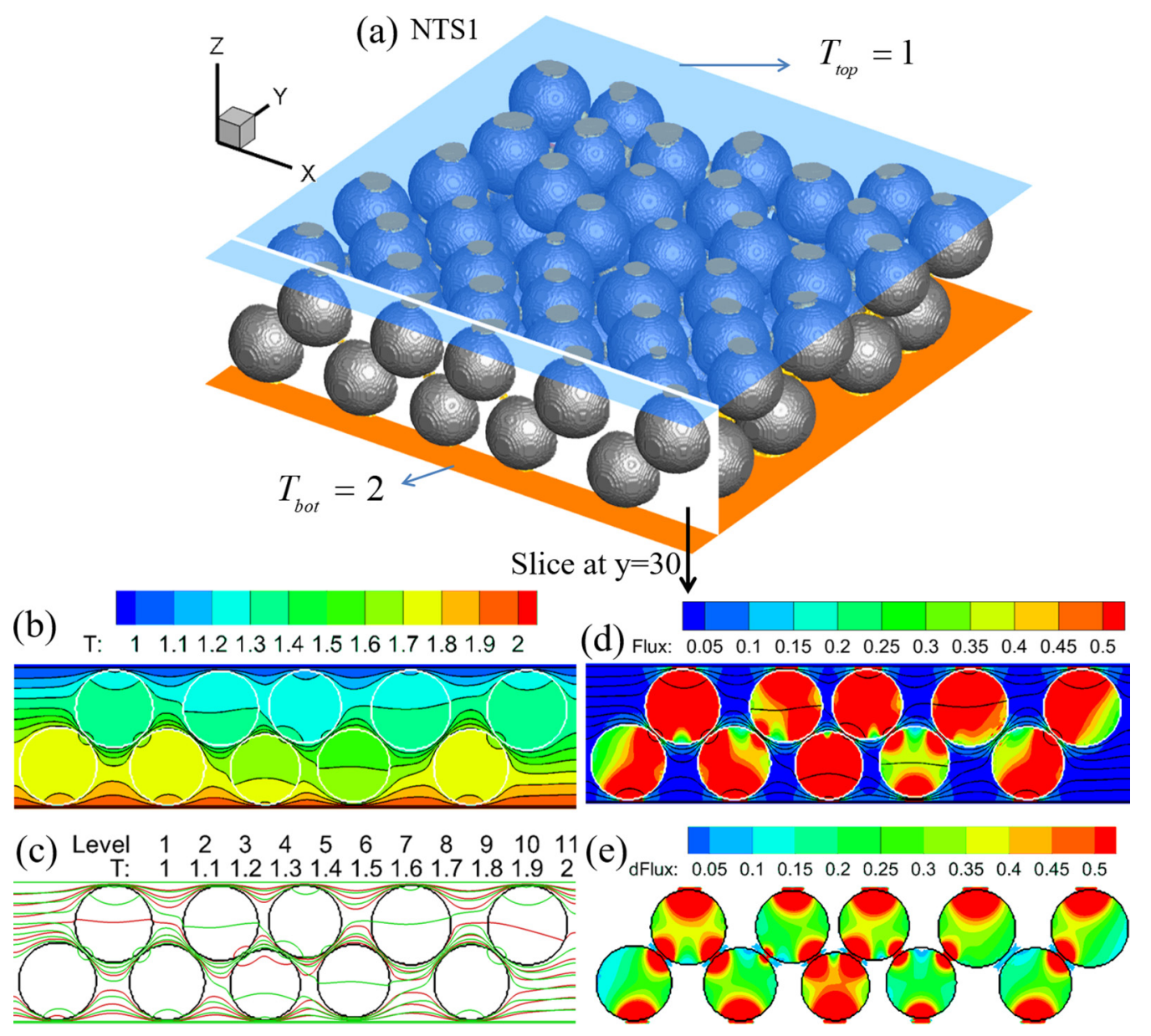

FIG. 11. Thermal performances of the neck-based thermal structure (NTS). (a) Simulation setup of NTS1 with the top and bottom temperatures $T_{\text {top }}=1$ and $T_{\text {bot }}=2$ and the four lateral sides adiabatic. (b) Temperature distribution with isolines (black) at the slice of $y=30$ and the white lines denoting the boundaries of the filler particles. (c) Comparison of temperature isolines between NTS1 (green lines) and PTS1 [red lines from Fig. 5(b)] at the slice of $y=30$, with black lines being boundaries of filler particles. (d) Heat flux distribution at the slice of $y=30$, with black lines being temperature isolines and white lines denoting the boundaries of filler particles. (e) Subtraction of heat flux distribution between NTS1 and PTS1 [from Fig. 5(c)] at the slice of $y=30$. All variables are in lattice units.

heat conduction, compared to what is observed in PTS1. The better heat conduction in NTS1 is also seen in Fig. 11(d) on the heat fluxes, which are higher in the filler particles as compared to the heat fluxes in PTS1 shown in Fig. 5(c). A subtraction of the heat fluxes between NTS1 and PTS1 at the slice of $y=30$ is shown in Fig. 11(e), illustrating that the heat conductions at the necks and filler particles around the necks are greatly enhanced in NTS. The temperatures and heat fluxes at the slice of $y=30$ in NTS2 and NTS3 are shown in Fig. S7 in the Supplemental Material.

The calculated ETCs for NTS1 to NTS3 are $\lambda_{\text {eff }}(\mathrm{NTSs})=$ $2.75,2.78,2.71 \mathrm{~W} / \mathrm{mK}$, respectively. Similar to PTSs, the small variation of $2.5 \%$ also indicates that the filler particle configurations have negligible influence on the overall thermal performance of NTSs, although the local temperature and heat flux are different depending on the distribution of filler particles. Compared to the experimental result of $2.41 \mathrm{~W} / \mathrm{mK}$, the simulated ETC has an overestimation of $14.1 \%$. The deviation is attributed to several possible reasons similar to those identified in the PTS evaluation. Specifically, the size of the sample may not be large enough. The adiabatic boundary condition at the four lateral sides may not take into account possible heat loss to the environment as occurs in the experiment. The void space between filler particles and nanoparticles may not be perfectly filled by epoxy. And neglecting the thermal contact resistance between filler particles/bottom/top and epoxy may overestimate the ETCs.

Finally, the overall enhancement of the heat conduction is assessed by comparing the ETCs of PTS and NTS between simulated results and also with experimental results. Figure 12 shows that, compared to PTS, the heat conduction of NTS is enhanced to 2.4 and 2.3 times in experimental and simulation results. The NTS is shown to be a good strategy to replace PTS between different layers of 3D chip stacks for an improved heat conduction. We note that, by comparing simulation results with experimental ones, the current combination of computational models provides valid results for the study the heat conduction in PTS and NTS of 3D chip stacks. 


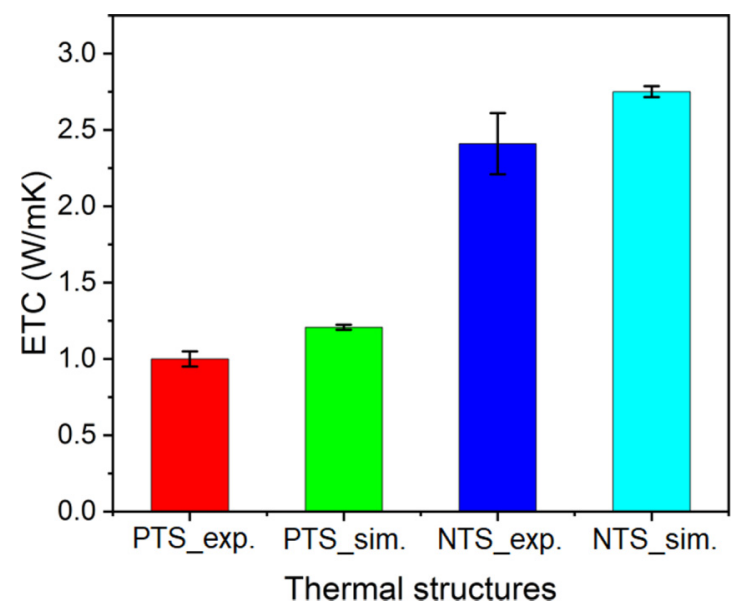

FIG. 12. Comparison of effective thermal conductivity (ETC) between PTS and NTS of both simulations and experiments.

\section{CONCLUSIONS}

As innovative techniques, the percolating and neck-based thermal structures (PTS, NTS) have been shown to enhance heat conduction in 3D chip stacks by two- to threefold experimentally. However, detailed explanations of the mechanisms of colloidal liquid drying, neck formation, and its influence on heat transport in multiscale porous structures have not been provided yet. In this paper, we numerically modeled the critical fabrication steps of PTS/NTS, evaluated their thermal performance of the different systems, and revealed the underlying mechanisms at play. First, we simulated the assembly of filler particles using discrete element method, to obtain three different filler particle configurations with very similar volume fraction. Then we evaluated the thermal performance of the resultant PTSs in terms of effective thermal conductivity (ETC) using a thermal LBM. Afterwards, using a tricoupled hybrid LBM, we simulated the neck formation by nanoparticle deposition after drying of colloidal suspension in the assembled filler particle configuration (FPC) to produce NTSs. Finally, we calculated the ETC of neck materials, using the methodology developed to asses PTS, and evaluated the thermal performance of NTSs, providing detailed analysis of temperature and heat flux at different locations.

The simulation results have shown that the drying process is continuous as the drying fronts recedes from the periphery to the center of the microporous structure. The drying rate demonstrates a decreasing trend with a turning point in the drying process. With regard to the neck formation, more necks with smaller size are observed between filler particles while fewer necks with larger size are seen at the top and bottom of the structures. Moreover, the neck count, size, and distribution are found not to be influenced by the filler particle configuration, when the filler particle volume fraction remains constant. Considering the heat conduction, the simulated ETCs of PTSs and NTSs are around 15\%-20\% higher than the experimental results, mainly due to neglecting thermal contact resistance. Compared to PTSs, the NTSs enhance the heat conduction by 2.4 times, which is found to be a result of the improved heat flux through the necks, i.e., critical throats. Similarly, the thermal performances of NTSs are not influenced by the FPCs at a constant filler particle volume fraction.

The numerical studies in this paper have shown the accuracy and capability of the tricoupled hybrid LBM and thermal LBM for modeling the drying of colloidal suspension and heat conduction in microporous structures. The coupled models can further be applied to study heat conduction enhancement in 3D chips in terms of the influences of nanoparticle concentration, drying temperature, chip surface wettability, and more, which are paths for future work. More generally, these models show high potentials to study other scientific/engineering problems related to drying of colloidal suspension and heat conduction, such as surface coating, salt transport/precipitation and food preserving.

\section{ACKNOWLEDGMENTS}

LANL's Institutional Computing Program and the Swiss National Super Computing Center (Project No. s823) are acknowledged for providing the computing support. Dr. O. Dorastkar is acknowledged for the assistance in modeling the assembly of filler particles. R. Fischer is acknowledged for helping to analyze the neck structures. The Swiss National Science Foundation (SNF), Project No. 160189 and No. 175793 is acknowledged for financial support.

F. Q., D. D., and J. C conceived the project. F. Q. performed the simulations and wrote the paper. All the authors analyzed the data and discussed the results. All authors have made comments and given approval to the final version of the manuscript.

The authors declare no competing financial interest.
[1] M. A. Boles, M. Engel, and D. V. Talapin, Chem. Rev. 116, 11220 (2016).

[2] M. Su, Z. Huang, Y. Li, X. Qian, Z. Li, X. Hu, Q. Pan, F. Li, L. Li, and Y. Song, Adv. Mater. 30, 1703963 (2018).

[3] M. Su, Z. Huang, Y. Huang, S. Chen, X. Qian, W. Li, Y. Li, W. Pei, H. Chen, F. Li, and Y. Song, Adv. Mater. 29, 1605223 (2017).

[4] C. Hamon, M. Postic, E. Mazari, T. Bizien, C. Dupuis, P. EvenHernandez, A. Jimenez, L. Courbin, C. Gosse, F. Artzner, and V. Marchi-Artzner, ACS Nano 6, 4137 (2012).

[5] S. Chen, M. Su, C. Zhang, M. Gao, B. Bao, Q. Yang, B. Su, and Y. Song, Adv. Mater. 27, 3928 (2015).

[6] J. Park and J. Moon, Langmuir 22, 3506 (2006).
[7] Z. Zhang, M. Su, Q. Pan, Z. Huang, W. Ren, Z. Li, Z. Cai, Y. Li, F. Li, L. Li, and Y. Song, ACS Appl. Mater. Interfaces 11, 1616 (2019).

[8] D. S. Bolintineanu, G. S. Grest, J. B. Lechman, F. Pierce, S. J. Plimpton, and P. R. Schunk, Comput. Part. Mech. 1, 321 (2014).

[9] T. Brunschwiler, J. Zürcher, L. Del Carro, G. Schlottig, B. Burg, S. Zimmermann, U. Zschenderlein, B. Wunderle, F. SchindlerSaefkow, and R. Stässle, J. Electron. Packag. 138, 041009 (2016).

[10] T. Brunschwiler, G. Schlottig, S. Ni, Y. Liu, J. V. Goicochea, J. Zürcher, and H. Wolf, Int. Symp. Microelectron. 2012, 000749 (2012). 
[11] J. Zurcher, X. Chen, B. R. Burg, S. Zimmermann, R. Straessle, A. R. Studart, and T. Brunschwiler, IEEE Trans. Components, Packag. Manuf. Technol. 6, 1785 (2016).

[12] See Supplemental Material at http://link.aps.org/supplemental/ 10.1103/PhysRevE.103.023311 for further information including the supplementary figures, table and animations.

[13] I. A. M. Elfadel and G. Fettweis, 3D Stacked Chips: From Emerging Processes to Heterogeneous Systems (Springer, Berlin, 2016).

[14] A. Srivastava, Introduction: Hardware and Software, in Academic Press Library in Signal Processing (Elsevier, Amsterdam, 2014), pp. 629-632.

[15] H. P. Huinink, L. Pel, M. A. J. Michels, and M. Prat, Eur. Phys. J. E 9, 487 (2002).

[16] N. Vorhauer, E. Tsotsas, and M. Prat, Phys. Rev. Fluids 3, 114201 (2018).

[17] M. Prat, Chem. Eng. J. 86, 153 (2002).

[18] I. U. Vakarelski, D. Y. C. Chan, T. Nonoguchi, H. Shinto, and K. Higashitani, Phys. Rev. Lett. 102, 058303 (2009).

[19] J. Perelaer, P. J. Smith, C. E. Hendriks, A. M. J. Van Den Berg, and U. S. Schubert, Soft Matter 4, 1072 (2008).

[20] S. A. Ryu, J. Y. Kim, S. Y. Kim, and B. M. Weon, Sci. Rep. 7, 1 (2017).

[21] D. Or, P. Lehmann, E. Shahraeeni, and N. Shokri, Vadose Zo. J. 12, 1 (2013).

[22] P. Coussot, Eur. Phys. J. B 15, 557 (2000).

[23] C. Chen, P. Duru, P. Joseph, S. Geoffroy, and M. Prat, Sci. Rep. 7, 15110 (2017).

[24] C. Chen, P. Joseph, S. Geoffroy, M. Prat, and P. Duru, J. Fluid Mech. 837, 703 (2018).

[25] P. Fantinel, O. Borgman, R. Holtzman, and L. Goehring, Sci. Rep. 7, 1 (2017).

[26] N. Shahidzadeh-Bonn, A. Azouni, and P. Coussot, J. Phys. Condens. Matter 19, 112101 (2007).

[27] N. Shokri and D. Or, J. Colloid Interface Sci. 391, 135 (2013).

[28] T. Pham and S. Kumar, Langmuir 33, 10061 (2017).

[29] F. Qin, A. M. Moqaddam, Q. Kang, D. Derome, and J. Carmeliet, Phys. Fluids 30, 032104 (2018).

[30] A. Mazloomi M., S. S. Chikatamarla, and I. V. Karlin, Phys. Rev. E 92, 023308 (2015).

[31] S. Son, L. Chen, D. Derome, and J. Carmeliet, Comput. Fluids 117, 42 (2015).

[32] A. Montessori, P. Prestininzi, M. La Rocca, and S. Succi, Phys. Fluids 29, 092103 (2017).

[33] X. Shan, Phys. Rev. E 55, 2780 (1997).

[34] I. V. Karlin, D. Sichau, and S. S. Chikatamarla, Phys. Rev. E 88, 063310 (2013).

[35] A. J. C. Ladd and R. Verberg, J. Stat. Phys. 104, 1191 (2001).

[36] U. Lantermann and D. Hänel, Comput. Fluids 36, 407 (2007).

[37] L. Chen, Q. Kang, Y. Mu, Y. L. He, and W. Q. Tao, Int. J. Heat Mass Transf. 76, 210 (2014).
[38] H. Liu, Q. Kang, C. R. Leonardi, S. Schmieschek, A. Narváez, B. D. Jones, J. R. Williams, A. J. Valocchi, and J. Harting, Comput. Geosci. 20, 777 (2016).

[39] J. Zhao, Q. Kang, J. Yao, H. Viswanathan, R. Pawar, L. Zhang, and H. Sun, Water Resour. Res. 54, 1295 (2018).

[40] M. Zhao and X. Yong, Phys. Rev. Fluids 3, 034201 (2018).

[41] Y. Ma, A. Bhattacharya, O. Kuksenok, D. Perchak, and A. C. Balazs, Langmuir 28, 11410 (2012).

[42] K. Yang and Z. Guo, Comput. Fluids 124, 157 (2016).

[43] F. Qin, A. M. Moqaddam, L. Del Carro, Q. Kang, T. Brunschwiler, D. Derome, and J. Carmeliet, Phys. Rev. E 99, 053306 (2019).

[44] F. Qin, A. Mazloomi Moqaddam, Q. Kang, D. Derome, and J. Carmeliet, LBM Simulation of self-assembly of clogging structures by evaporation of colloidal suspension in $2 \mathrm{D}$ porous media, Transp. Porous Media 128, 929 (2019).

[45] M. Wang, J. Wang, N. Pan, and S. Chen, Phys. Rev. E 75, 036702 (2007).

[46] M. Wang, Q. Kang, and N. Pan, Appl. Therm. Eng. 29, 418 (2009).

[47] C. Xie, J. Wang, D. Wang, N. Pan, and M. Wang, Commun. Comput. Phys. 17, 1037 (2015).

[48] W. Z. Fang, J. J. Gou, H. Zhang, Q. Kang, and W. Q. Tao, Numer. Heat Transf. Part A Appl. 70, 1101 (2016).

[49] M. R. Sridhar and M. M. Yovanovich, J. Thermophys. Heat Transfer 8, 633 (1994).

[50] M. Bahrami, J. R. Culham, M. M. Yananovich, and G. E. Schneider, Appl. Mech. Rev. 59, 1 (2006).

[51] X. Chen and P. Han, Int. J. Heat Fluid Flow 21, 463 (2000).

[52] F. Qin, L. Del Carro, A. M. Moqaddam, Q. Kang, T. Brunschwiler, D. Derome, and J. Carmeliet, J. Fluid Mech. 866, 33 (2019).

[53] I. V Karlin, A. Ferrante, and H. C. Öttinger, Europhys. Lett. 47, 182 (1999).

[54] I. V. Karlin, F. Bösch, and S. S. Chikatamarla, Phys. Rev. E 90, 031302 (2014).

[55] M. Sbragaglia, R. Benzi, L. Biferale, S. Succi, K. Sugiyama, and F. Toschi, Phys. Rev. E 75, 026702 (2007).

[56] P. Yuan and L. Schaefer, Phys. Fluids 18, 042101 (2006)..

[57] D. M. Anderson, G. B. McFadden, and A. A. Wheeler, Annu. Rev. Fluid Mech. 30, 139 (1998).

[58] Q. Li, Q. J. Kang, M. M. Francois, and A. J. Hu, Soft Matter 12, 302 (2016).

[59] L. K. Limbach, Y. Li, R. N. Grass, T. J. Brunner, M. A. Hintermann, M. Muller, D. Gunther, and W. J. Stark, Environ. Sci. Technol. 39, 9370 (2005).

[60] O. Dorostkar, R. A. Guyer, P. A. Johnson, C. Marone, and J. Carmeliet, Geophys. Res. Lett. 44, 6101 (2017).

[61] O. Dorostkar, R. A. Guyer, P. A. Johnson, C. Marone, and J. Carmeliet, J. Geophys. Res. Solid Earth 122, 3689 (2017). 